\title{
2d Frictional B-Spline Smoothed Mortar Contact Problems Part II: Resolution Phase
}

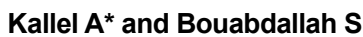

Pôle Universitaire Léonard De Vinci, De Vinci Reserach Center, Paris La Défense, France

Laboratoire Roberval UMR 7337, Université Technologique de Compiègne, 60205 Compiègne, France

\begin{abstract}
We detailed in this paper three formulations for the resolution of a contact problem by mortar method. The penalty method is a simple technique which does not introduce new unknowns which can increase the size of the system to be solved. But this formulation suffers of conditioning problems especially when the penalty coefficient becomes very high. The Lagrange multipliers method is more accurate than the penalty formulation. The multiplier $\lambda_{N}$ represents in the contact surface the exact value of the normal contact effort. This approach requires additional variables which are the Lagrange multiplier in the contact interface nodes. The augmented Lagrange method is a combination between the penalty formulation and the Lagrange multipliers method. The contact constraints are applied by a Lagrange multiplier approached without increasing the problem size. The penalty coefficient in this method has less influence on the quality of the result and the robustness of the solution than in the penalty formulation.
\end{abstract}

\section{Keywords: Resolution phase; Mortar; Resolution}

\section{Introduction}

In literature, there are various methods for applying the contact constraints and modelling its contribution to the overall solution of the problem [1-5]. In this work, we focus on three methods: Penalty, Lagrange multipliers and Augmented Lagrange.

The penalty method is still the most used in industrial computer codes. It transforms the contact conditions written as an inequality in a system of equations by adding a penalty term noted $\varepsilon_{N}$. This technique is simple to implement and interpret the physical point of view. Contact constraints are verified exactly that for a penalty coefficient tends to infinity. A very high value of this coefficient leads to a poor conditioning system to solve. A low value results in a violation of the conditions of non-penetration. The quality of the solution obtained by this method strongly depends on the choice of the value of the penalty coefficient.

In the Lagrange multiplier method, the contact conditions are exactly verified by the introduction of additional degrees of freedom called Lagrange multipliers [3,6,7]. This formulation should be applied in combination with an activation strategy (commonly called Active Set Strategy) which controls and updates in each convergence loop multipliers assets and liabilities [8,9]. Only active multipliers are among the unknowns of the problem. If the conditions for non-penetration are raped in a non-mortar segment (slave), every point of the latter integration will contribute to the contact force in order to increase the contact stiffness and reduce the penetration to zero. Lagrange multipliers associated with the node of this element will be activated. This condition is applied as a contact initialization as the item is still in touch since its Lagrange multipliers are disabled by default. The elements already in contact, their average multipliers integration point must be negative for the multipliers remain active node. If the contact stress in a non-mortar segment becomes positive, this segment turn into inactive status and its associated Lagrange multipliers disappear and will be removed from the system to solve. For this reason, the two multipliers same element or mortar segment not always have the same status: either active or inactive. The Lagrange multiplier method does not allow penetration relative to the start of contact activation. Unlike penalty method, the contact conditions in this method apply a brutal way [10]. This makes this method less robust than the penalty method because of convergence difficulties in some cases.
The method of Augmented Lagrange is a kind of formulation with Lagrange multipliers regularized by a penalty coefficient. This method can exactly meet the contact constraints without over forcing the issue with additional unknowns. The method of the augmented Lagrange satisfies the conditions of non-penetration progressively starting from a zero-initial value of the multiplier and increasing its amplitude as a function of the penetration value. Powell [11] used for optimization problems, an algebraic formulation to update the Lagrange multipliers at each iteration. Today, this approach is known as the algorithm name Uzawa. Another approach has been developed in [12] and it consists on the minimization of a continuous problem (saddle point) with simultaneous updating of primal and dual variables. Glowinski and Le Tallec [13] presented one of the first applications of the Lagrange method increased at a frictionless contact problem. A generalization of the method by Uzawa algorithm to frictional problems was developed in $[5,14]$. Augmented Lagrange formulation with finite element method for contact problems with friction in large displacement is implemented in [15]. The method of Augmented Lagrange combines the benefits of both a penalty and Lagrange multipliers while avoiding their disadvantages. It converges to the solution for a large penalty coefficient without influencing the proper conditioning of the problem. This technique requires an additional update algorithm, but the computational effort required by this algorithm is reasonable. The Lagrange multipliers method does not write the optimization problem with inequality constraints as an unconstrained problem since the Lagrange multipliers of positivity condition $\lambda_{N} \geq 0$ must be satisfied. The method of Augmented Lagrange does not have this restriction and it is therefore preferable for practical use.

*Corresponding author: Kallel A, Pôle Universitaire Léonard de Vinci, De Vinci Reserach Center, Paris La Défense, France, Tel: +33 1411670 00; E-mail: achraf.kallel@devinci.fr

Received June 14, 2017; Accepted June 30, 2017; Published July 04, 2017

Citation: Kallel A, Bouabdallah S (2017) 2d Frictional B-Spline Smoothed Mortar Contact Problems Part II: Resolution Phase. J Appl Mech Eng 6: 275. doi: 10.4172/2168-9873.1000275

Copyright: ( 2017 Kallel A, et al. This is an open-access article distributed under the terms of the Creative Commons Attribution License, which permits unrestricted use, distribution, and reproduction in any medium, provided the original author and source are credited. 
The non-differentiability of the normal contact force and term of the friction (for frictional problem) presents a major problem for the numerical resolution of the contact problem. The contribution of the contact in the formulation of the whole problem can be written thanks to various techniques. In the literature, a large part of mechanical problems adopts the Lagrange multipliers technique to guarantee the continuity of the displacement field between both domains in the interface. To formulate the contact problem with this method, a Lagrange multiplier $\lambda$ can be regarded as a contact pressure in the interface $\delta g_{N}$. This multiplier is an unknown of the problem brought by the slave surface. The Lagrange multiplier method applies strictly the Signorini conditions. The brutality of this application makes the calculation difficult to converge. The Lagrange multipliers are considered as additional degrees of freedom at each node of the slave area, thus increasing the size of the problem to solve.

The penalty method is also ubiquitous in the literature for the modeling of contact problems. The principle of this method is to introduce a penalty coefficient to progressively apply Signorini terms (Figure 1).

\section{Linearization of Contact Problem Variables}

Owed in geometrical not linearity, the variation of the normal gap $\delta g_{N}$ and the tangential gap $\delta g_{T}$ in the contact interface $\Gamma_{\mathrm{c}}$ has to be linearized.

\section{Frictionless contact}

For frictionless contact problem, it is necessary to give an expression of the displacement field according to the normal gap linearization $\Delta g_{N}$ and its variation $\Delta \delta g_{N^{*}}$. The normal gap expression is given by:

$$
g_{N}=\left[x^{n m}-x^{m}(\xi)\right] \cdot n^{m}(\xi) \geq 0
$$

The variation of (1) gives:

$$
\delta g_{N=} \delta\left(\left[x^{n m}-x^{m}(\xi)\right] \cdot n^{m}(\xi)\right)
$$

The expression of (2) in the mortar contact interface can be written as:

$$
\delta g_{N^{\prime}=}\left[\delta x^{n m}-\delta x^{m}(\xi)\right] . n^{m}(\xi)+\left[x^{n m} x^{m}(\xi)\right] . \delta n^{m}(\xi)
$$

The distance which separates both bodies is computed by using the vector $\mathbf{g}=\overline{x^{m m} x^{m}}$. This value is minimal when this vector becomes collinear to the normal vector of the mortar surface $n^{m}$. We can deduct that $n^{m} . \delta n^{m}=0$ for the value of $\xi$ which fulfils this condition. We can rewrite (3) as follows:

$$
\delta g_{N}=\left[\delta x^{n m}-\delta x^{m}(\xi)\right] \cdot n^{m}(\xi)
$$

We replace $\delta x^{n m}$ by $\Delta u^{n m}$ and $\delta x^{m}$ by $\Delta u^{m}$; we can rewrite (1.4):

$\Delta \mathrm{g}_{N}=\left[\Delta u^{n m}-\Delta u^{m}(\xi)\right] \cdot n^{m}(\xi)$

Let write the expression (1) otherwise:

$x^{n m}-x^{m}(\xi)=g_{N} n^{m}$

The variation of (6) gives:

$$
\delta x^{n m}-\delta x^{m}(\xi)-x_{, \xi}^{m} \delta \xi=\delta g_{N} n^{m}+g_{N} \delta n^{m}
$$

The vector $x^{n m}$ does not depend on the natural coordinate $\xi$, while the displacement field and the vector $x^{m}$ depend on this variable. The linearization of (7) gives:

$$
\begin{aligned}
& -\left[\delta x_{, \xi}^{m} \Delta \xi+\Delta u_{, \xi}^{m} \delta \xi+x_{, \xi \xi}^{m} \Delta \xi \delta \xi+x_{, \xi}^{m} \Delta \delta \xi\right] \\
& =\Delta\left(\delta g_{N}\right) n^{m}+\delta g_{N} \Delta n^{m}+\Delta g_{N} \delta n^{m}+g_{N} \Delta \delta n^{m}
\end{aligned}
$$

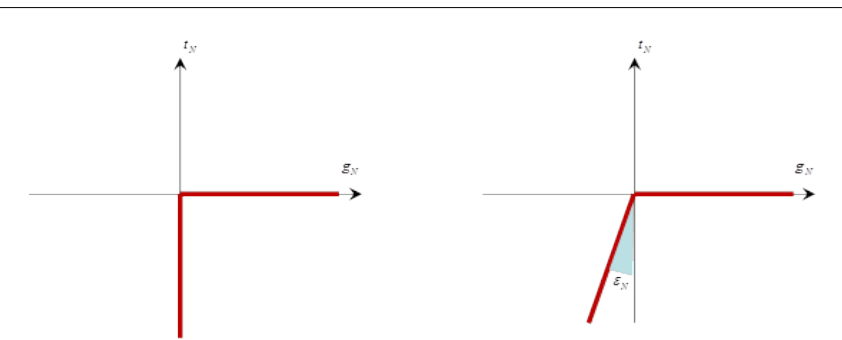

Figure 1: Application of the Signorini conditions: Left with the Lagrange multipliers formulation and right with penalty method.

The displacement field is interpolated with a linear shape function. The vector which describes the position of a point on the mortar surface $x^{m}$ is then linear on $\xi$. The second derivative this vector is zero $x_{, \xi \xi}^{m}=0$. The value of $\xi$ indicates the position of the mortar point $x^{m}$ in the mortar segment between nodes $N_{i}^{m}$ and $x_{1}^{m}$. Let suppose that the position of these two nodes correspond respectively to points $x_{1}^{m}$ and $x_{2}^{m}$. We can then define a tangent vector on the mortar surface $a^{m}=x_{2}^{m}-x_{1}^{m}$. The normal vector for the mortar surface is a function of the natural coordinate $\xi$. If the point $x^{m}$ becomes the closest mortar point to the non-mortar point $x^{n m}$, the orthogonality conditions between and will be verified $a^{m}$ and $n^{m}$ will be fulfils and we can write:

$\delta n^{m} \cdot a^{m}=-n^{m} \cdot \delta a^{m}$

Knowing that $n^{m}$. $\delta n^{m}=0$, we can express the variation of the normal vector as follows:

$$
\delta n^{m}=-\left[a^{m} \otimes n^{m}\right] \cdot \delta a^{m}=-\left(n^{m} \cdot \delta a^{m}\right) \cdot a^{m}
$$

And its linearization gives:

$\Delta n^{m}=-\left[a^{m} \otimes n^{m}\right] \cdot \Delta a^{m}=-\left(n^{m} \cdot \Delta a^{m}\right) \cdot a^{m}$

For the point $x^{m}$ which verifies the projection condition, the natural coordinate can be expressed as follows:

$$
\xi=\frac{\left(x^{n m}-x_{1}^{m}\right) \cdot\left(x_{2}^{m}-x_{1}^{m}\right)}{\left(x_{2}^{m}-x_{1}^{m}\right) \cdot\left(x_{2}^{m}-x_{1}^{m}\right)}
$$

And its variation:

$$
\delta \xi=\frac{1}{\left\|x_{2}^{m}-x_{1}^{m}\right\|^{2}}\left[\left(\delta x^{n m}-\delta x^{m}\right) \cdot x_{, \xi}^{m}+g_{N} n^{m} \cdot \delta x_{, \xi}^{m}\right]
$$

For the linearization of (13) we can write:

$$
\Delta \xi=\frac{1}{\left\|x_{2}^{m}-x_{1}^{m}\right\|^{2}}\left[\left(\Delta u^{n m}-\Delta u^{m}\right) \cdot a^{m}+g_{N} n^{m} \cdot \Delta u_{, \xi}^{m}\right]
$$

If we multiply the equality ( 8 ) by the normal vector $n^{m}$, we obtain:

$$
\begin{aligned}
& -\left[\delta x_{, \xi}^{m} \Delta \xi+\Delta u_{, \xi}^{m} \delta \xi+x_{, \xi \xi}^{m} \Delta \xi \delta \xi+x_{, \xi}^{m} \Delta \delta \xi\right] \cdot n^{m} \\
& =\Delta\left(\delta g_{N}\right)+\delta g_{N} \Delta n^{m} \cdot n^{m}+\Delta g_{N} \delta n^{m} \cdot n^{m}+g_{N} \Delta \delta n^{m} \cdot n^{m}
\end{aligned}
$$

As $n^{m} \cdot \delta n^{m}=0$ and $n^{m} \cdot \Delta n^{m}=0$, we can rewrite (1.15):

$\Delta\left(\delta g_{N}\right)=-\left[\delta x_{, \xi}^{m} \Delta \xi+\Delta u_{, \xi}^{m} \delta \xi+x_{, \xi \xi}^{m} \Delta \xi \delta \xi+x_{, \xi}^{m} \Delta \delta \xi\right] \cdot n^{m}-g_{N} \Delta \delta n^{m} \cdot n^{m}$

By using these relations:

$$
\begin{aligned}
& n^{m} . \delta n^{m}=0 \\
& \Delta\left(n^{m} \cdot \delta n^{m}\right)=0 \\
& \Delta n^{m} . \delta n^{m}+n^{m} \cdot \Delta \delta n^{m}=0 \\
& n^{m} . \Delta \delta n^{m}=-\Delta n^{m} . \delta n^{m}
\end{aligned}
$$




$$
\Delta\left(\delta g_{N}\right)=-\left[\delta x_{, \xi}^{m} \Delta \xi+\Delta u_{, \xi}^{m} \delta \xi+x_{, \xi \xi}^{m} \Delta \xi \delta \xi+x_{, \xi}^{m} \Delta \delta \xi\right] \cdot n^{m}+g_{N} \Delta n^{m} \cdot \delta n^{m}
$$

By using the expression of linearized normal vector (11), we can write:

$$
\Delta\left(\delta g_{N}\right)=-\left(\delta x_{, \xi}^{m} \Delta \xi+\Delta u_{, \xi}^{m} \delta \xi\right) \cdot n^{m}+\frac{g_{N}}{\left\|x_{2}^{m}-x_{1}^{m}\right\|^{2}}\left(n^{m} \cdot \delta x_{, \xi}^{m}\right) \cdot\left(\Delta u_{, \xi}^{m} \cdot n^{m}\right)
$$

\section{Frictional contact}

We handle the linearization of the tangential contact variables according to the displacement fields of both bodies in contact with friction. If we have friction, it is necessary to distinguish the stick contact of the slip contact. We have to see again the expression (14) as well as the linearization of (13). The expression (8) was multiplied by the normal vector in the case of frictionless contact to find a linearization of the variation of the natural coordinate $\Delta \delta \xi$. In the case of a tangent contact we multiply this expression by the tangent vector $a^{m}$.

$$
\begin{aligned}
& -\left[\delta x_{, \xi}^{m} \Delta \xi+\Delta u_{, \xi}^{m} \delta \xi+x_{, \xi \xi}^{m} \Delta \xi \delta \xi+x_{, \xi}^{m} \Delta \delta \xi\right] \cdot a^{m} \\
& =\Delta\left(\delta g_{N}\right) n^{m} \cdot a^{m}+\delta g_{N} \Delta n^{m} \cdot a^{m}+\Delta g_{N} \delta n^{m} \cdot a^{m}+g_{N} \Delta \delta n^{m} \cdot a^{m}
\end{aligned}
$$

By applying the orthogonality condition $n^{m} \cdot a^{m}=0$ and by using a linear interpolation we can rewrite (20) as follows:

$$
-x_{, \xi}^{m} \cdot a^{m} \Delta \delta \xi=\delta x_{, \xi}^{m} \Delta \xi+\Delta u_{, \xi}^{m} \delta \xi+\delta g_{N} \Delta n^{m} \cdot a^{m}+\Delta g_{N} \delta n^{m} \cdot a^{m}+g_{N} \Delta \delta n^{m} \cdot a^{m}
$$

The tangent vector can be defined by $\left\|x_{2}^{m}-x_{1}^{m}\right\|^{2}$. The coefficient before $\Delta \delta \xi$ in (21) present its dot product which is equal to $\left\|x_{2}^{m}-x_{1}^{m}\right\|^{2}$. By using these equations

$$
\begin{aligned}
\Delta\left[\delta\left(n^{m} \cdot a^{m}\right)\right] & =\Delta\left[\delta n^{m} \cdot a^{m}+n^{m} \cdot \delta a^{m}\right] \\
& =\Delta \delta n^{m} \cdot a^{m}+\delta n^{m} \cdot \Delta a^{m}+\Delta n^{m} \cdot \delta a^{m}+n^{m} \cdot \Delta \delta a^{m}=0
\end{aligned}
$$

We can write

$$
\Delta \delta n^{m} \cdot a^{m}=-\left(\delta n^{m} \cdot \Delta a^{m}+\Delta n^{m} \cdot \delta a^{m}+n^{m} \cdot \Delta \delta a^{m}\right)
$$

Let us resume the variation of the gap in (2) and let us calculate its linearization:

$$
\begin{aligned}
& {\left[\delta x^{n m}-\delta x^{m}(\xi)\right]=g_{N} \delta n^{m}} \\
& {\left[\Delta u^{n m}-\Delta u^{m}(\xi)\right]=g_{N} \Delta n^{m}}
\end{aligned}
$$

In (21), one already knows the expression of $\delta \xi$ and of $\Delta \xi$, for the other three terms one use (23) and (24) then we can write:

$$
\delta g_{N} \Delta n^{m} \cdot a^{m}+\Delta g_{N} \delta n^{m} \cdot a^{m}-g_{N}\left(\delta n^{m} \cdot \Delta a^{m}+\Delta n^{m} \cdot \delta a^{m}\right)=\left(\delta x^{m m}-\delta x^{m}\right) \cdot \Delta a^{m}-\left(\Delta u^{m m}-\Delta u^{m}\right) \cdot \delta a^{m}
$$

We can then give an expression for the linearization of the variation of $\xi$ :

$$
\Delta \delta \xi=\frac{1}{\left\|x_{2}^{m}-x_{1}^{m}\right\|^{2}}\left[-2 \cdot a^{m}\left(\Delta u_{, \xi}^{m} \delta \xi+\delta x_{, \xi}^{m} \Delta \xi\right)+\left(\delta x^{m m}-\delta x^{m}\right) \cdot \Delta u_{, \xi}^{m}+\left(\Delta u^{m m}-\Delta u^{m}\right) \cdot \delta x_{, \xi}^{m}\right]
$$

\section{Frictionless contact}

Penalty term reacts as a spring situated at the contact interface preventing the interpenetration of the two bodies. Although present in many industrial codes, this technique is sensitive to the value of the penalty coefficient.

A combination of these two techniques has led to the formulation of contact with Augmented Lagrange. The Lagrange multipliers in this method no longer presented as additional unknown to the problem. This technique ensures, in addition, not larger load compared with the two previous methods.

\section{Penalty method}

In this approach, the normal component of the contact force is proportional to the value of the normal gap:

$$
\lambda_{N}=\varepsilon_{N} g_{N}
$$

One study the expression of $C_{N}$ in the case of the penalty method, the contact contribution to the virtual work on a non-mortar segment can be written as follows:

$$
G_{c}=\int_{\Gamma_{c}} \varepsilon_{N} g_{N} \delta g_{N} d \Gamma=\varepsilon_{N} \sum_{G P=1}^{n_{G P}} g_{N}(\zeta) \delta g_{N}(\zeta)\left\|X_{2}^{n m}-X_{1}^{n m}\right\| \omega_{G P}
$$

With $n_{G P}$ is the number of the active integration points into the considered non-mortar segment. $\omega_{G P}$ refers to the integration weight associated with the point and $\left\|x_{2}^{\prime \prime}-x_{-1}^{\prime \prime}\right\|$ the initial length of the segment (also denoted $l_{0}^{n m}$ ). Since the variables with this method are the gap and its variation, the linearization of $C_{N}$ gives:

$$
\frac{\partial c_{N}}{\partial \lambda_{N}}=0, \quad \frac{\partial c_{N}}{\partial g_{N}}=\varepsilon_{N} \delta g_{N}, \quad \frac{\partial c_{N}}{\partial \delta g_{N}}=\varepsilon_{N} g_{N}
$$

With:

$\Delta c_{N}=\varepsilon_{N} \delta g_{N} \Delta g_{N}+\varepsilon_{N} g_{N} \Delta \delta g_{N}$

To satisfy the conditions of non-penetration, a contact pressure, denoted $p_{N}$, is created on the contact interface when the gap becomes negative (Figure 2).

With the penalty formulation, the Hertz-Signorini-Moreau conditions are not satisfied in the strict sense, since the contact pressure is generated only if there is penetration between the two bodies (Figure 2 ). The normal contact pressure $p_{N}$ is presented in $[2,16,17]$ as a linear relationship between the normal gap $g_{N}$ and a penalty coefficient noted $\varepsilon_{N}$. The normal pressure and the gap are proportional; they are linked by the relationship

$$
p_{N}=\varepsilon_{N} g_{N}
$$

The more the contact effort brought up, the more the penetration decrease and the Hertz-Signorini-Moreau conditions satisfied. This approximation implies that the contact surface does not eliminate penetration, but resists penetration.

The physical interpretation leads to a representation of the contact surface as a series of springs with zero initial length which may stretch out inside the body perpendicular to the main surface. The reaction shows a linear relationship with the spring extension. The extension for the spring is equivalent to the penetration for a contact problem with the penalty formulation. To minimize this elongation (penetration), we must maximize the stiffness of the spring (penalty coefficient). Too high values of $\varepsilon_{N}$, lead to a bad conditioning of the system to solve and a degradation of the convergence.

\section{Expressions of the Residual Vector and the Tangent Ma- trix}

One considers a non-mortar segment $s_{j}^{m}$ in contact with a mortar segment $s_{j}^{m}$. The integration points in $s_{j}^{n m}$ are projected onto $s_{j}^{n m}$. Due to the non-conforming of the mesh, integration points in $s_{j}^{n m}$ can be projected on mortar segments neighbors of $s_{i-1}^{m}$ as $s_{i-1}^{m}$ or $s_{i+1}^{m}$. The expression of the normal gap (6) applied to integration point $x_{G P}^{n m}(\zeta)$ is given by:

$$
g_{N}=\left[x_{G P}^{n m}(\zeta)-\bar{x}^{m}(\bar{\xi})\right] \cdot n^{m}(\bar{\xi})
$$

To calculate the normal gap $g N$ and its variation $\delta g_{N}$, we have first to calculate the natural coordinated $\xi$ for each projection. From (1) and 


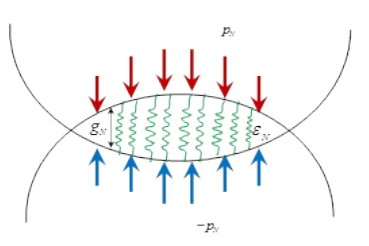

Figure 2: Interpenetration of the springs in the interface: Penalty method (83)

using the projection condition $n^{m} \cdot a^{-m}=0$, the projection may result in the following scalar product:

$$
\left(x_{G P}^{n m}-\bar{x}^{m}\right) \cdot a^{m}=0
$$

With $a^{m}$, is the tangent vector on the mortar segment containing the projection (Figure 3 ). Its expression is as follows:

$$
a^{m}=x_{2}^{m}-x_{1}^{m}
$$

The position of the projection point $x^{-m}$ in a mortar segment is given, depending on the position of two nodes of the segment $x_{1}^{m}$ and $x_{2}^{m}$ by:

$$
\bar{x}^{m}=(1-\bar{\xi}) x_{1}^{m}+\bar{\xi} x_{2}^{m}
$$

Similarly, the non-mortar point to be projected $x_{1}^{n m}$ is connected to both non-mortar nodes $x_{1}^{n m}$ and $x_{2}^{n m}$ by:

$$
x_{G P}^{n m}=(1-\zeta) x_{1}^{n m}+\zeta x_{2}^{n m}
$$

Expression (2.6) may be described as follows:

$$
x_{G P}^{n m} \cdot a^{m}-x_{1}^{m} \cdot a^{m}+\bar{\xi}\left(x_{1}^{m}-x_{2}^{m}\right) \cdot a^{m}=0
$$

Then

$$
x_{G P}^{n m} \cdot a^{m}-x_{1}^{m} \cdot a^{m}+\bar{\xi}\left(x_{1}^{m}-x_{2}^{m}\right) \cdot a^{m}=0
$$

The expression of the natural coordinated $\bar{\xi}$ required for the calculation of the gap is given by:

$$
\bar{\xi}=\frac{\left(x_{G P}^{n m}-x_{1}^{m}\right) \cdot a^{m}}{a^{m} \cdot a^{m}}
$$

Using (34) and (35) in the expression of the gap (31), one obtains:

$$
g_{N}=\left[(1-\zeta) x_{1}^{n m}+\zeta x_{2}^{n m}-(1-\xi) x_{1}^{m}-\xi x_{2}^{m}\right] \cdot \bar{n}^{m}(\xi)
$$

Using a matrix writing more suited to the algorithmic implementation, we can write:

$$
\left\langle x_{n}\right\rangle=\left\langle\left\langle x_{1}^{m m}\right\rangle,\left\langle x_{2}^{m m}\right\rangle,\left\langle x_{1}^{m}\right\rangle,\left\langle x_{2}^{m}\right\rangle\right\rangle
$$

With:

$$
\begin{aligned}
& \left\langle x_{n}\right\rangle=\left\langle\left\langle x_{1}^{n m}\right\rangle,\left\langle x_{2}^{n m}\right\rangle,\left\langle x_{1}^{m}\right\rangle,\left\langle x_{2}^{m}\right\rangle\right\rangle \\
& \left\{n^{m}\right\}=\left\{\begin{array}{l}
n_{x}^{m} \\
n_{y}^{m}
\end{array}\right\} \\
& B_{G P}=\left[\begin{array}{l}
N_{1}^{n m}(\zeta) \cdot \hat{1} \\
N_{2}^{n m}(\zeta) \cdot \hat{1} \\
-N_{1}^{m}(\xi) \cdot \hat{1} \\
-N_{2}^{m}(\xi) \cdot \hat{1}
\end{array}\right] \text { Which } \quad \hat{1}=\left[\begin{array}{ll}
1 & 0 \\
0 & 1
\end{array}\right]
\end{aligned}
$$

The variation of the expression (31) gives:

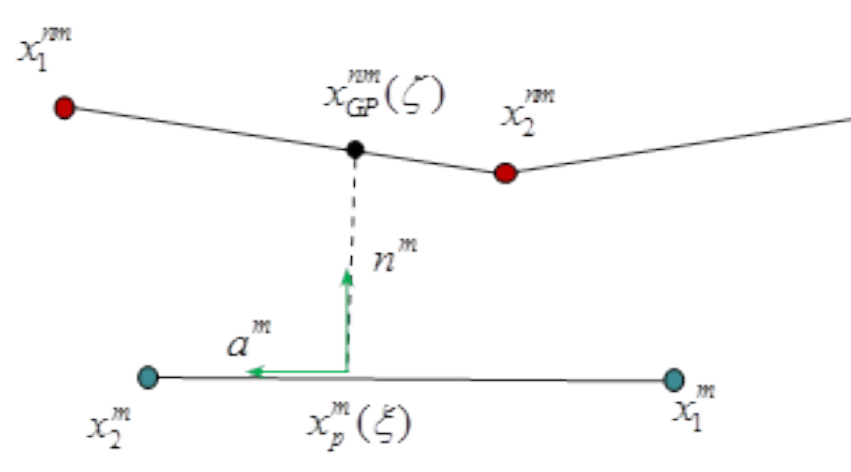

Figure 3: Projection into the contact mortar interface.

$$
\delta g_{N}=\left[\delta x_{G P}^{n m}(\zeta)-\delta \bar{x}^{m}(\bar{\xi})\right] \cdot n^{m}(\bar{\xi})
$$

In matrix form:

$$
\begin{aligned}
& \delta g_{N}=\left\langle\delta x_{n}\right\rangle\left[B_{G P}\right]\left\{n^{m}\right\} \\
& \text { Avec }\left\langle\delta x_{n}\right\rangle=\left\langle\left\langle\delta x_{1}^{n m}\right\rangle,\left\langle\delta x_{2}^{n m}\right\rangle,\left\langle\delta x_{1}^{m}\right\rangle,\left\langle\delta x_{2}^{m}\right\rangle\right\rangle
\end{aligned}
$$

We can rewrite (28) as follows:

$$
\left.G_{c}=\int_{\Gamma_{c}} \varepsilon_{N} g_{N} \delta g_{N} d \Gamma=\varepsilon_{N} \sum_{G P=1}^{n_{G P}}\left[\left\langle\delta x_{n}\right\rangle\left(\left[B_{G P}\right]\left\{n^{m}\right\}\left\langle n^{m}\right\rangle\left[B_{G P}\right]^{T}\right)\left\{x_{n}\right\}\right]\right]_{0}^{n m} \omega_{G P}
$$

The contact residual vector for penalty formulation can be written as follows:

$$
R_{N}^{P e n}=\varepsilon_{N}\left[\left[B_{G P}\right]\left\{n^{m}\right\}\left\langle n^{m}\right\rangle\left[B_{G P}\right]^{T}\right]\left\{x_{n}\right\} l_{0}^{n m} \omega_{G P}
$$

It remains to linearize the second term in (30) to obtain the tangent matrix expression. From (35) and (38), and by using $a^{m}=x_{. \xi}^{m}$, the variation of the natural coordinate $\xi$ is given by:

$$
\begin{aligned}
& \delta \xi=\frac{1}{\left(l^{m}\right)^{2}}\left[\left(\delta x^{n m}-\delta \bar{x}^{m}\right) \cdot \bar{x}_{, \xi}^{m}+g_{N} \bar{n}^{m} \cdot \delta \bar{x}_{, \xi}^{m}\right] \\
& \text { Which } l^{m}=\left\|a^{m}\right\|
\end{aligned}
$$

In the mortar surface we can note ${ }^{m m}\left(X^{m m}, t\right)=X^{m m}+u^{m m}\left(X^{n m}, t\right.$ and in the non-mortar surface we can note $x^{m m}\left(X^{n m}, t\right)=X^{n m}+u^{n m}\left(X^{n m}, t\right)$. Using these two relations we can write:

$$
\Delta \xi=\frac{1}{\left(l^{m}\right)^{2}}\left[\left(\Delta u^{n m}-\Delta u^{m}\right) \cdot \bar{x}_{, \xi}^{m}+g_{N} n^{m} \cdot \Delta u_{, \xi}^{m}\right]
$$

The linearization of (2.18) gives:

$$
\Delta g_{N}=\left[\Delta u^{n m}-\Delta u^{m}(\xi)\right] \cdot n^{m}(\xi)
$$

Finally, the linearization of the variation of the gap $\Delta\left(\delta g_{N}\right)$ in the normal direction can be written as:

$$
\Delta\left(\delta g_{N}\right)=-\left(\delta x_{, \xi}^{m} \Delta \xi+\Delta u_{, \xi}^{m} \delta \xi\right) \cdot \bar{n}^{1}+\frac{g_{N}}{\left(l^{m}\right)^{2}} \delta x_{, \xi}^{m} \cdot\left[n^{m} \otimes n^{m}\right] \Delta u_{, \xi}^{m}
$$

Substituting expressions (49) and (50) in (52) we get:

$$
\begin{aligned}
\Delta\left(\delta g_{N}\right)= & -\left(\delta a^{m}\left[\frac{1}{\left(l^{m}\right)^{2}}\left[\left(\Delta u^{u m}-\Delta u^{m}\right) \cdot a^{m}+g_{N} n^{m} \cdot \Delta u_{, \xi}^{m}\right]\right]\right. \\
& \left.+\Delta u_{, \xi}^{m}\left[\frac{1}{\left(l^{m}\right)^{2}}\left[\left(\delta x^{m m}-\delta \bar{x}^{m}\right) \cdot a^{m}+g_{N} \bar{n}^{m} \cdot \delta a^{m}\right]\right]\right) \cdot \bar{n}^{1} \\
& +\frac{g_{N}}{\left(l^{m}\right)^{2}} \delta a^{m} \cdot\left[n^{m} \otimes n^{m}\right] \Delta u_{, \xi}^{m}
\end{aligned}
$$


We know that $x^{n m}\left(X^{n m}, t\right)=X^{n m}+u^{n m}\left(X^{n m}, t\right)$ and that $n^{m} \cdot \bar{a}^{m}=0,(53)$

can be described as follows:

$$
\begin{aligned}
\Delta\left(\delta g_{N}\right)= & -\Delta \xi\left(\delta x_{2}^{m}-\delta x_{1}^{m}\right) \cdot n^{m}+\left[\delta x^{m m}-(1-\bar{\xi}) \delta x_{1}^{m}-\bar{\xi} \delta x_{2}^{m}\right] \cdot n^{m} \\
= & -\frac{1}{l^{m}}\left[\Delta u^{m m}-(1-\bar{\xi}) \Delta u_{1}^{m}-\bar{\xi} \Delta u_{2}^{m}\right] \cdot a^{m}\left(\delta x_{2}^{m}-\delta x_{1}^{m}\right) \cdot n^{m} \\
& -\frac{g_{N}}{\left(l^{m}\right)^{2}}\left(\delta x_{2}^{m}-\delta x_{1}^{m}\right) \cdot n^{m}\left(\Delta u_{2}^{m}-\Delta u_{1}^{m}\right) \cdot n^{m} \\
& -\frac{1}{l^{m}}\left[\delta x^{m m}-(1-\bar{\xi}) \delta x_{1}^{m}-\bar{\xi} \delta x_{2}^{m}\right] \cdot a^{m}\left(\Delta u_{2}^{m}-\Delta u_{1}^{m}\right) \cdot n^{m}
\end{aligned}
$$

Using (34), (39) and (51), the expression (54) becomes:

$$
\begin{aligned}
\Delta\left(\delta g_{N}\right)=\left\langle\delta x_{n}\right\rangle & {\left[-\frac{1}{\left(l^{m}\right)^{2}}\left(\left[B_{G P, \xi}\right]\left\{n^{m}\right\rangle\left\langle a^{m}\right\rangle\left[B_{G P}\right]^{T}+\left[B_{G P}\right]\left\{a^{m}\right\}\left\langle n^{m}\right\rangle\left[B_{G P, \xi}\right]^{T}\right.\right.} \\
& \left.\left.+g_{N}\left[B_{G P, \xi}\right]\left\{n^{m}\right\}\left\langle n^{m}\right\rangle\left[B_{G P, \xi}\right]^{T}\right)\right]\left\{\Delta x_{n}\right\}
\end{aligned}
$$

In matrix form we can rewrite (30) as follows:

$$
\Delta\left(c_{N}\right)=\left\langle\delta x_{n}\right\rangle\left[K_{N}^{P e n}\right]\left\{\Delta x_{n}\right\}
$$

Finally, the tangent matrix for the penalty formulation is given by:

$$
\begin{aligned}
& K_{N}^{P_{N o n}}=\varepsilon_{N}\left[\left[B_{G P}\right]\left\{n^{m}\right\}\left\langle m^{m}\right\rangle\left[B_{G P}\right]^{T}-\left(\left[B_{G P, i}\right]\left\{n^{m}\right\}\left\langle a^{m}\right\rangle\left[B_{G P}\right]^{T}+\left[B_{G P}\right]\left\{a^{m}\right\}\left\langle n^{m}\right\rangle\left[B_{G P, \xi}\right]^{T}\right.\right. \\
& \left.\left.+g_{N}\left[B_{G P, \xi}\right]\left\{n^{m}\right\rangle\left\langle n^{m}\right\rangle\left[B_{G P, \xi}\right]^{T}\right) \frac{g_{N}}{\left\|a^{m}\right\|^{2}}\right] l_{0}^{l^{m m}} \omega_{G P}
\end{aligned}
$$

Where,

$$
\begin{aligned}
& \left\{a^{m}\right\}=\left\{\begin{array}{l}
a_{x}^{m} \\
a_{y}^{m}
\end{array}\right\} \\
& \left\langle\Delta x_{n}\right\rangle=\left\langle\left\langle\Delta x_{1}^{n m}\right\rangle,\left\langle\Delta x_{2}^{n m}\right\rangle,\left\langle\Delta x_{1}^{m}\right\rangle,\left\langle\Delta x_{2}^{m}\right\rangle\right\rangle \\
& B_{G P, \xi}=\left[\begin{array}{c}
\hat{0} \\
\hat{0} \\
-N_{1, \xi}^{m}(\xi) \cdot \hat{1} \\
-N_{2, \xi}^{m}(\xi) \cdot \hat{1}
\end{array}\right] \text { which } \hat{0}=\left[\begin{array}{ll}
0 & 0 \\
0 & 0
\end{array}\right] \text { and } \hat{0}=\left[\begin{array}{ll}
0 & 0 \\
0 & 0
\end{array}\right]
\end{aligned}
$$

The residual vector (48) and the tangent matrix (57) present the contribution of an integration point in the contact interface (Figure 3 ) to the global system. Note that we have to take into account the contribution of all the integration points of the interface.

\section{Resolution Algorithm}

At each load step, the contact constraints are considered only for actives integration points. In the penalty method, an integration point is considered active if the normal gap calculated at this point is negative or zero (contact or interpenetration). If in the next load step, a point which was active finds itself with a strictly positive gap, it does not contribute any more to the calculation of the residual vector and of the tangent matrix (Figure 4).

Note that in the same segment, certain integration points can be active while others will not. The contribution to the calculation of contact forces is treated point by point for this method. After resolution of the nonlinear system obtained by the NewtonRaphson, the contact pressure is determined at each integration point by the equation:

$$
P_{N}=\varepsilon_{N} g_{N}
$$

A detailed resolution algorithm for the penalty method is shown in Figure 5.

\section{Validation Example}

An aluminum beam is half placed on a steel support. To prevent its tipping, a tray with $15 \mathrm{~mm}$ long is placed on the beam from the right end to prevent the vertical movement. A vertical force is applied downward on the left end of the beam (Figures 6 and 7).

$$
\begin{aligned}
& \text { Material characteristics: } \\
& E_{\text {support }}=2.1 \times 10^{11} \mathrm{pa} \\
& v_{\text {support }}=0.3 \\
& E_{\text {beam }} 6.9 \times 10^{9} \mathrm{pa} \\
& V_{\text {beam }}=0.33 \\
& \text { Loading: }
\end{aligned}
$$$$
\mathrm{F}_{\mathrm{y}=} 1.0 \times 10^{8} \mathrm{~N}
$$

Note the influence of the penalty coefficient on the quality of the solution (Figure 8). For this example of contact between the steel and aluminum, the value $\varepsilon_{N}=1 \times 10^{10}$ was necessary to avoid overlap between the beam and the support at the sharp angle. The reaction in the horizontal direction is stabilizing from the value of $\varepsilon_{N}=1 \times 10^{9}$, whereas a value from $\varepsilon_{N}=1 \times 10^{10}$ is required to stabilize the vertical reactions (Figure 8). The coefficient of penalty can be estimated according to the rigidity of bodies in contact as it can be updated during the calculation according to the penetration [18].

\section{Lagrange Multipliers Method}

We can also transform the writing of a contact problem described by an inequality to an equations system (equalities) by using Lagrange multipliers. This method initially used in the theory of the optimization to find a minimal value of a function submitted to different constraints. For the contact problem formulation, the constraints to be verified are the conditions of non-penetration. The Lagrange multipliers represent the pressure contacts which are an unknown to calculate in an optimized way to satisfy these conditions. This problem, in certain circumstances, can be replaced by the search for a fixed point of the saddle point of a function which the unknown is the Lagrange multiplier. Contact constraints enforcement with the Lagrange multipliers formulation is developed in this work with the method mortar for the discretization of the contact interface. This method allows the coupling of sub domains stemming from contact areas of both bodies defined by two different meshes.

The Lagrange multipliers will be considered as additional unknowns in the system to be solved. For a modelling with the finite element method, it means that these multipliers will be treated as degrees of

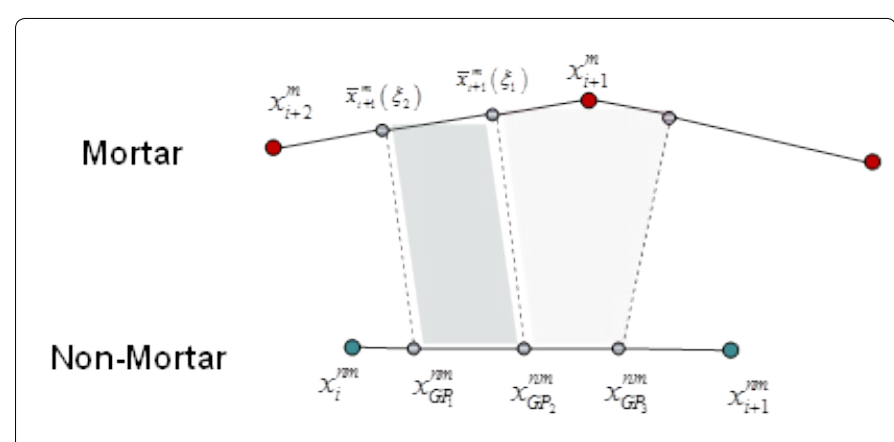

Figure 4: Segmentation of the mortar contact interface with the penalty method. 


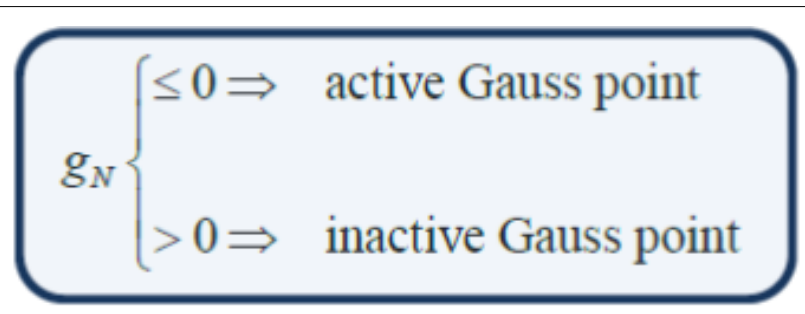

Figure 5: Activation condition of the integration points for the penalty method.

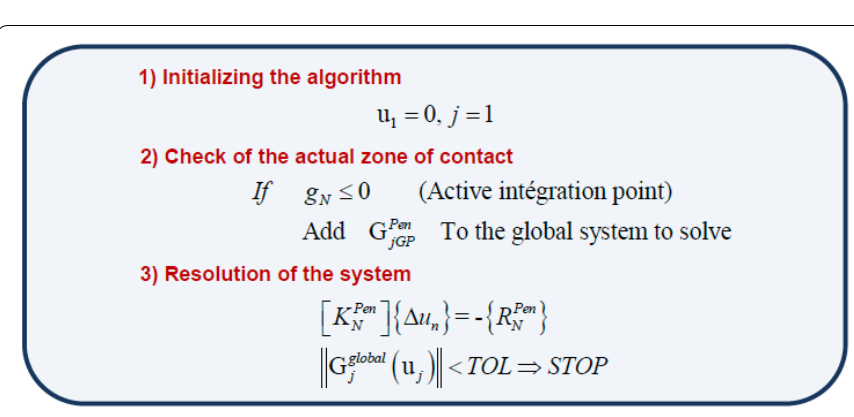

Figure 6: Algorithm of resolution of the contact problem using penalty method.

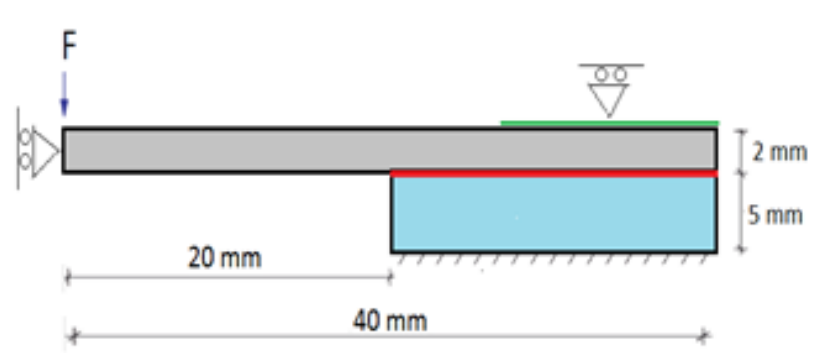

Figure 7: Contact problem between a beam and a support.

freedom furthermore which establish a field to be calculated with the displacement field. As for the interpolation of the displacement field, it is necessary to use shape functions for the interpolation of the Lagrange multipliers. It remains now to clarify the choice of these functions. The choice of the shape functions for the interpolation of the Lagrange multipliers is very important. As presented in [5], we chose first the following shape functions:

$$
M_{1}^{n m}=2-3 \zeta \quad M_{2}^{n m}=-1+3 \zeta
$$

Both expressions of $M_{i}^{n m}$ and of $M_{i}^{n m}$ are a function of the natural coordinate $\zeta$ seen that the Lagrange multipliers are defined on nonmortar segments (slave surface). In the literature, we can find a dual application of the Lagrange multipliers which consists in succeeding the integration of the multipliers on the slave surface then on the master surface or the invers. We can find more detail of this technique in the works of [19-26]. The shape functions adopted by Wohlmouth [19] and presented in (62) do not express a maximal value of the multiplier in the extremities. If we replace the value of the natural coordinate $\zeta$ by 0 and by 1 , the shape functions do not nullify. Then, the maximal value of the multiplier is inside the element and not in the summit (Figure 9).

The shape functions for the Lagrange multipliers $M_{i}^{n m}$ have to be of the same order as those used for the interpolation of the displacement field. After testing the shape functions proposed by Wolmuth [19], we finally opted, in this work, for the use of identical shape functions for the interpolation of the displacement field and the Lagrange multipliers. With this choice, the Lagrange multipliers are defined on nodes and they will be considered as an additional degree of freedom.

In this approach, the normal component of the effort is equal to the normal Lagrange multiplier $\lambda_{N}$. We define the expression of the contact contribution to the virtual work on a non-mortar segment in the case of the Lagrange multipliers formulation, and then we can write:

Since that the variables in this method are the normal gap $g_{N}$ and its variation $\delta g_{N}$ as well as the normal multiplier $\lambda_{N}$ and its variation $\delta \lambda_{N}$, the linearization of $C_{N}$ is translated by:

$$
\begin{aligned}
& \Delta c_{N}=\delta g_{N} \Delta \lambda_{N}+\delta \lambda_{N} \Delta g_{N}+\lambda_{N} \Delta \delta g_{N} \\
& \text { And } \\
& \Delta c_{N}=\delta g_{N} \Delta \lambda_{N}+\delta \lambda_{N} \Delta g_{N}+\lambda_{N} \Delta \delta g_{N}
\end{aligned}
$$

The Lagrange multiplier injected in the solution represents physically the contact pressure. Its rough introduction in the contact formulation returns the resolution more difficult than a problem approached by the penalty method in which the answer is progressive and proportional to the penetration. The solution of the problem requires the use of the Newton-Raphson method. The tangent matrix and the residual vector for the Lagrange multipliers formulation will be detailed in the section which follows.

\section{Expressions of the Residual Vector and the Tangent Matrix}

We take back the expression of the gap in (39) and we write it under matrix shape in the case of the Lagrange multipliers formulation:

$$
\left\langle x_{n}^{L a g}\right\rangle=\left\langle\left\langle x_{1}^{m m}\right\rangle,\left\langle\left\langle x_{2}^{m m}\right\rangle,\left\langle x_{1}^{m}\right\rangle,\left\langle x_{2}^{m}\right\rangle, \lambda_{N_{1}}, \lambda_{N_{2}}\right\rangle\right.
$$

When:

$$
\begin{aligned}
& \left\langle x_{n}^{L a g}\right\rangle=\left\langle\left\langle x_{1}^{n m}\right\rangle,\left\langle x_{2}^{n m}\right\rangle,\left\langle x_{1}^{m}\right\rangle,\left\langle x_{2}^{m}\right\rangle, \lambda_{N_{1}}, \lambda_{N_{2}}\right\rangle \\
& B_{G P}^{L a g}=\left[\begin{array}{c}
N_{1}^{n m}(\zeta) \cdot \hat{1} \\
N_{2}^{n m}(\zeta) \cdot \hat{1} \\
-N_{1}^{m}(\xi) \cdot \hat{1} \\
-N_{2}^{m}(\xi) \cdot \hat{1} \\
0 \\
0
\end{array}\right] \text { with }\left\langle x_{n}^{L a g}\right\rangle
\end{aligned}
$$

The vector $\left\langle x_{n}^{\text {Lag }}\right\rangle$ contains, compared with its equivalent in the penalty formulation, two additional unknowns $\lambda_{N_{1}}$ and $\lambda_{N_{2}}$ which correspond to the values of the Lagrange multiplier in the non-mortar segment nodes (Figure 10).

The variation of the expression (39) under matrix form for the Lagrange multipliers method is given by:

$$
\begin{aligned}
& \delta g_{N}=\left\langle\delta x_{n}\right\rangle\left[B_{G P}\right]\left\{n^{m}\right\} \\
& \text { With }\left\langle\delta x_{n}\right\rangle=\left\langle\left\langle\delta x_{1}^{n m}\right\rangle,\left\langle\delta x_{2}^{n m}\right\rangle,\left\langle\delta x_{1}^{m}\right\rangle,\left\langle\delta x_{2}^{m}\right\rangle, \delta \lambda_{N_{1}}, \delta \lambda_{N_{2}}\right\rangle
\end{aligned}
$$

The value of the Lagrange multiplier in an integration point belonging to a non-mortar segment is given by:

$\lambda_{N_{G P}}=(1-\zeta) \lambda_{1}+\zeta \lambda_{2}$

And under matrix shape: 

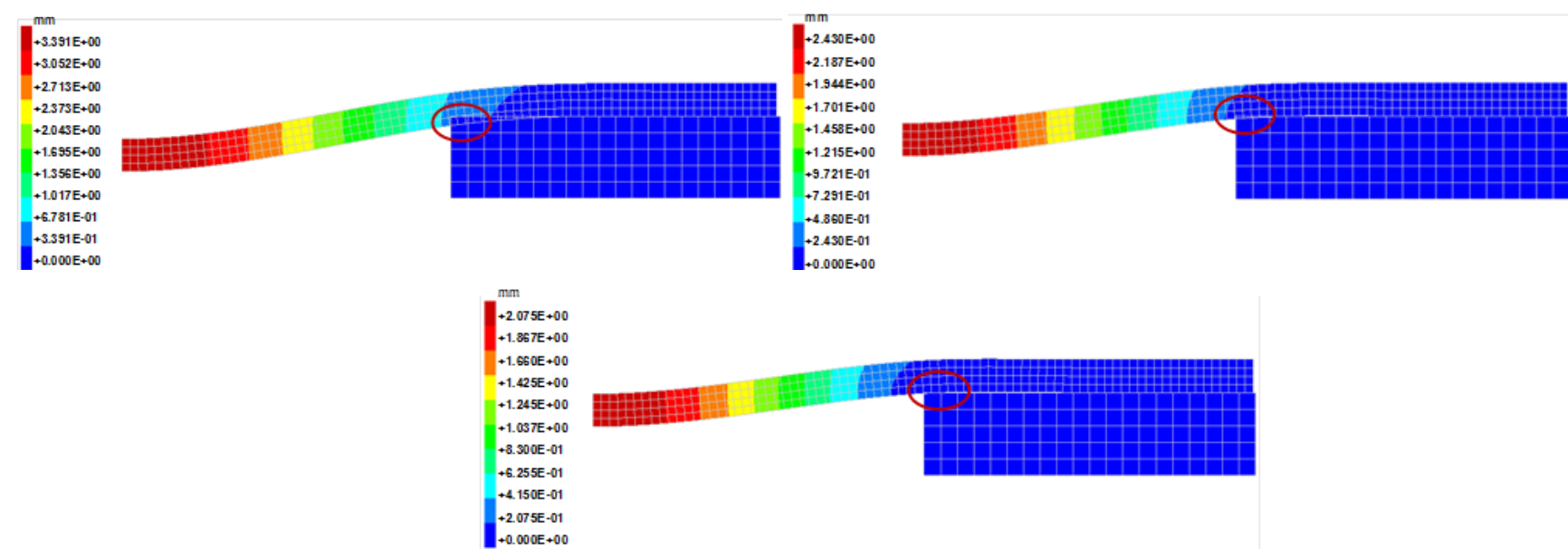

(a) Penalty coefficient $\varepsilon_{N}=1 \times 10^{8}$. (b) Penalty coefficient $\varepsilon_{N}=1 \times 10^{9}$. (c) Penalty coefficient $\varepsilon_{N}=1 \times 10^{10}$.

Figure 8: Deformed configuration of the contact problem between beam and support.

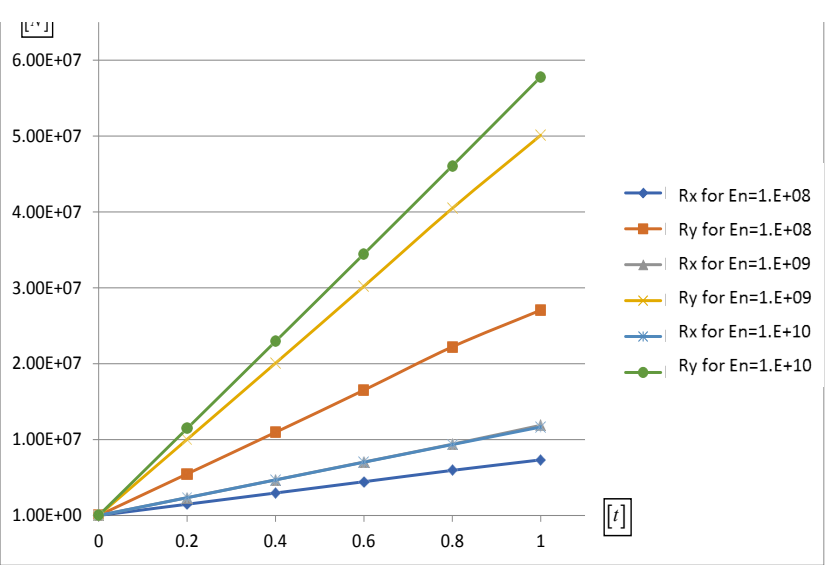

Figure 9: Variation of the normal and tangential reaction according to the value of the penalty coefficient.

$$
\lambda_{N_{G P}}=\left\langle x_{n}\right\rangle\left[M_{G P}^{L a g}\right]
$$

With

$$
\left[M_{G P}^{L p g}\right]=\left[\begin{array}{l}
0 \\
0 \\
0 \\
0 \\
0 \\
0 \\
0 \\
0 \\
M_{1}^{m m}(\zeta) \\
M_{2}^{m m}(\zeta)
\end{array}\right]
$$

The variation of $(72)$ is given by:

$$
\mathrm{T} \delta \lambda_{N_{G P}}=\left\langle\delta x_{n}\right\rangle\left[M_{G P}^{\text {Lag }}\right]
$$

We can rewrite (63) as follows:

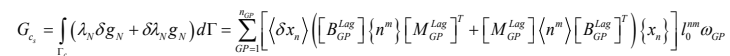

The residual vector for the contact can be written in the case of the Lagrange multipliers formulation as follows:

$$
{ }_{N}^{L a g}=\left[\left[B_{G P}^{L a g}\right]\left\{n^{m}\right\}\left[M_{G P}^{L a g}\right]^{T}+\left[M_{G P}^{L a g}\right]\left\langle n^{m}\right\rangle\left[B_{G P}^{L a g}\right]^{T}\right]\left\{x_{n}\right\} l_{0}^{n m} \omega_{c}
$$

To obtain the expression of the tangent matrix, it is necessary to linearize all the three terms of (65) and to rewrite it under matrix shape. With (51), (69) and (74), we can rewrite the first two terms of (65). It remains to find a matrix writing of the third term.

The variation of the natural coordinate $\xi$ is detailed in (49) and its linearization in (50). The linearization of the variation of the gap is given in (56).

Under matrix shape we can rewrite (2.39) as follows:

$$
\Delta\left(c_{N}\right)=\left\langle\delta x_{n}\right\rangle\left[K_{N}^{\operatorname{Lag}}\right]\left\{\Delta x_{n}\right\}
$$
by:

The tangent matrix for the Lagrange multiplier formulation is given

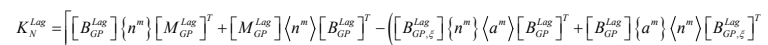

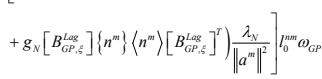

With

$$
B_{G P, \xi}^{L a g}=\left[\begin{array}{c}
\hat{0} \\
\hat{0} \\
-N_{1, \xi}^{m}(\xi) \cdot \hat{1} \\
-N_{2, \xi}^{m}(\xi) \cdot \hat{1} \\
0 \\
0
\end{array}\right]
$$

The residual vector residue (76) the tangent matrix (78) constitutes the contribution of an integration point in the contact interface (Figure 10) to the global system. Let us note that it is necessary to take into account the contribution of the all integration points in the interface.

\section{Algorithm of Resolution}

A non-mortar segment possesses one value of Lagrange multiplier at each node (Figure 8). Two multipliers of the same segment possess the same status: they are either active or inactive. The multipliers of every segment are inactive by default. A violation of the non-penetration 
condition activates the multiplier. The check of this condition is not made point by point as it is the case in the penalty method but rather on the entire segment. At every step load, the contact constraints are considered only for the active segments.

In the first load step, we verify the status of every segment. If the sum of gap weighted in the integration points of the same segment is negative, this segment becomes active and its multipliers will be considered in the system to be solved (Figure 11).

During the next step, the algorithm presented in the Figure 11 will be used for the inactive segments, but we add another algorithm to verify if the already active multipliers are going to maintain their status for this load step. The active segments keep their status if the sum of the weighted multipliers calculated in the integration points is negative. If this sum becomes positive, both nodal Lagrange multipliers of this segment will be deactivated and removed from the system to be solved. All the integration points of the segment will be, also, removed from the list of the active points, and they do not contribute any more to the calculation of the residual vector and of the tangent matrix (Figure 12).

A detailed resolution algorithm for the Lagrange multiplier method is shown in Figure 13.

\section{Example of Validation}

In this problem of contact with large displacement [20-27], a solid disk interacts with a half-crown (basis) with a vertical translation $\Delta_{\mathrm{v}}=80$ $U L$ (Figure 14). The radios of the disk and the contact surface of the basis is $\mathrm{R}=50 \mathrm{UL}$, whereas the external radios of the basis is $R_{e}=125 \mathrm{UL}$. A of Neo-Hookeen hyper-elastic behavior is considered for the material for the both solids. The disk, with a Young modulus of $\lambda_{N}^{(k+1)}=\left\langle\lambda_{N}^{(k)}+\varepsilon_{N} g_{N}\right\rangle$ , is 10 times stiffener than the basis. Both bodies present the same Poisson coefficient of $v=0.3$.

Materials characteristics:

$E_{\text {disk }}=1.10^{5} \mathrm{~F} / U L^{2}$

$v=0.3$

$E_{\text {basis }} 1.10^{4} \mathrm{~F} / \mathrm{UL}^{2}$

$v=0.3$

Loading:

$\Delta_{\mathrm{y}}=80 U L$

This example is similar to that of [28], in which Fischer and al used up to 20 points of integration by element. In our example, we noticed that we don't need to use more than 6 integration points to reach a good precision.

We notice that with the Lagrange multipliers method, the entire of the effort applied to the disk is taken back by the contact efforts (Figures 15-17). The sum of the latter is exactly equal for this formulation to the sum of the value of the Lagrange multipliers. What is not still the case with the method of penalty (as shown later).

\section{Augmented Lagrange Method}

This formulation aims at settling differentiability of contact terms (displacement and forces) for a problem with or without friction by combining the penalty method and the Lagrange multipliers method. This type of formulation was considered initially in the context of the stresses incompressibility [13], then in the frictionless contact problems [4] and [2], and finally for the resolution of the frictional contact

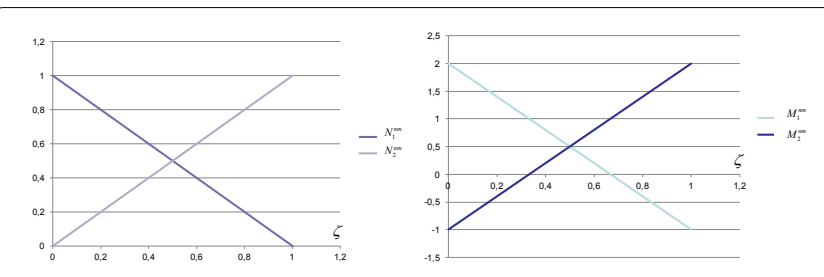

Figure 10: Shape functions for the interpolation of the Lagrange multipliers.

Mortar

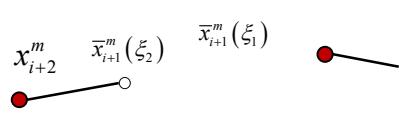

Non-mortar

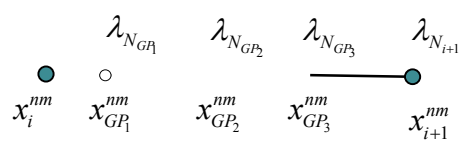

Figure 11: Segmentation of the mortar contact interface in the Lagrange multipliers method.

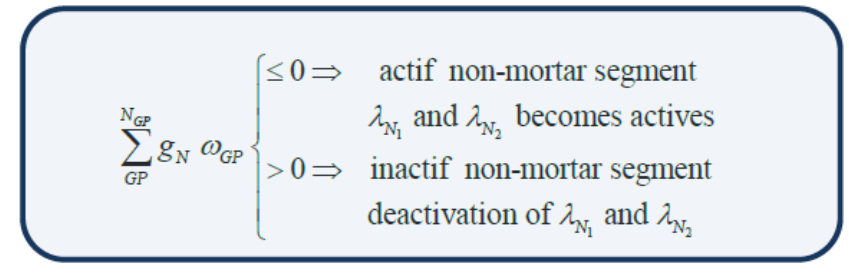

Figure 12: Activation of multipliers condition for the Lagrange method.

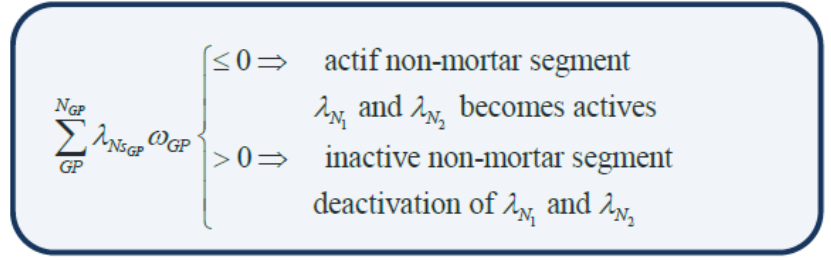

Figure 13: Check of the non-penetration condition and the preservation of the active integration points.

problems in large transformations as in $[5,14]$. This method leads to a $\mathrm{C}^{1}$ differentiable saddle-point problem [15].

The normal contact effort is obtained by the combination of a Lagrange multiplier and a penalty term. We look for a good approximation of the exact multiplier by this formula:

$$
\lambda_{N}^{(k+1)}=\left\langle\lambda_{N}^{(k)}+\varepsilon_{N} g_{N}\right\rangle
$$

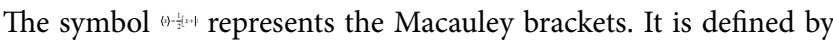
$\langle x\rangle=\frac{1}{2}[x+|x|] z$ and the index $\mathrm{k}$ presents the counter of the augmentations. A simplified variation of this expression through a technique adapted by Lagrange multipliers is used to update the value of the multipliers from iteration to the following one. The exact value of the Lagrange multiplier is obtained by an iterative algorithm of augmentation. This value is maintained during the newton iteration ' $i$ ' to solve the weak form and will be updated in the following iteration ' $i+1$ '. This algorithm 


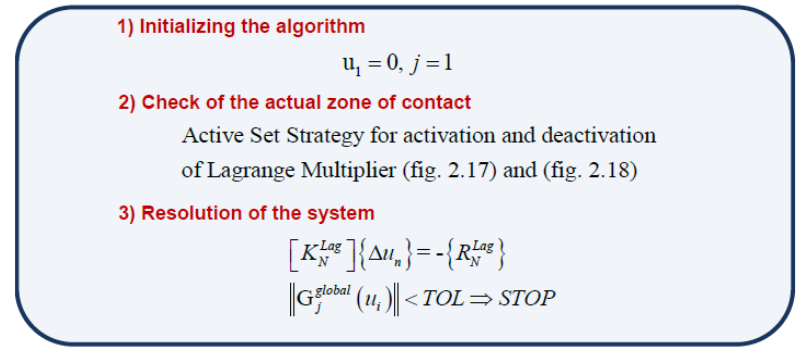

Figure 14: Resolution algorithms for the Lagrange multiplier method.

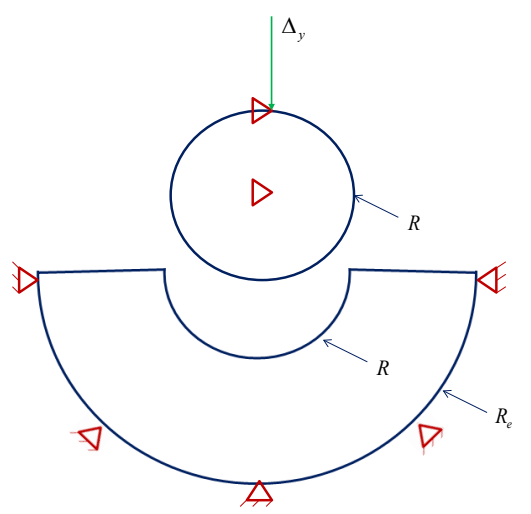

Figure 15: Data for the contact problem between disk and basis.
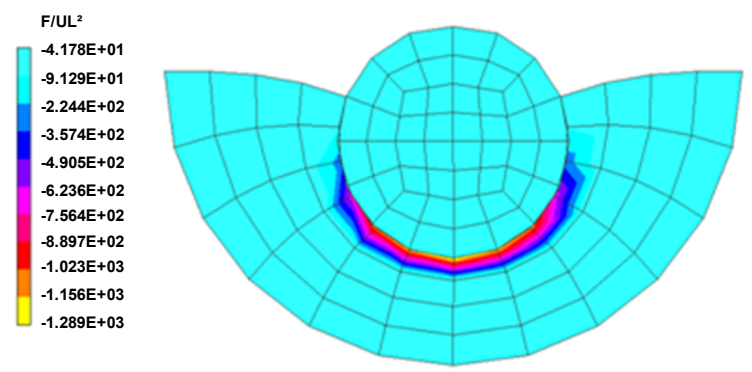

Figure 16 : Distribution of the Lagrange multipliers.
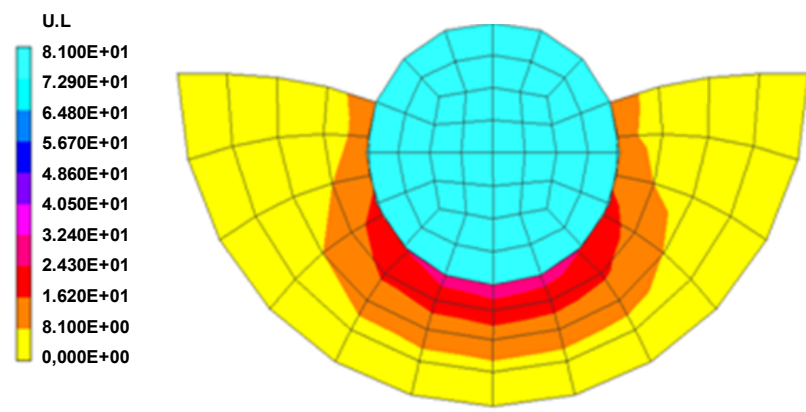

Figure 17: Displacement for the contact problem between disk and basis. of augmentation is known under the name of the technique of Uzawa (Figure 18).

For a point given in the interface, we try to calculate the normal contact effort for an active segment. An iterative process is necessary to reach the exact value of the Lagrange multiplier $\lambda_{N}$. At the beginning of every load step, the initial value of the Lagrange multiplier $\lambda_{N_{0}}$ of is null. The approximation of the contact effort will consist only of the penalty term. If the rigidity of contact at this stage is insufficient to satisfy the non-penetration conditions, a value of penetration is then detected. This value triggers the augmentation algorithm of the Lagrange multiplier.

The amplitude of the normal contact pressure before the first augmentation iteration is equal to the Lagrange multiplier $\lambda_{(1)}^{(n)}$ and which presents only the product of the penetration value by the penalty coefficient. The value of the multiplier obtained will be accumulated with the product of the new value of penetration and the penalty coefficient. This accumulation increases the value of the Lagrange multiplier from iteration to another one while the value of the penetration, it, decreases. The system to be solved will contain the new value of the multiplier. The value of the penetration continues to decrease with the iterations of augmentation until which nullifies (Figure 19). In this case (null penetration) the non-penetration condition will be verified and the value of the augmented multiplier obtained represents the exact value of the contact pressure. The Lagrange multiplier augmentation technique during a load step is detailed in the following scheme.

In this approach, the normal component of the effort is equal to the normal Lagrange multiplier $\lambda_{N}$ given by (80). We resume the expression of the of the normal contact contribution $C_{N}$ for the augmented Lagrange method, the contribution of the contact to the virtual work on a non-mortar segment can be written as follows:

$$
G_{c_{s}}=\int_{\Gamma_{c}}\left(\bar{\lambda}_{N}+\varepsilon_{N} g_{N}\right) \delta g_{N} d \Gamma=\sum_{G P=1}^{n_{C P}}\left(\left(\bar{\lambda}_{N}(\zeta)+\varepsilon_{N} g_{N}(\zeta)\right) \delta g_{N}(\zeta)\right) l_{0}^{n m} \omega_{G P}
$$

The variables with this method are the normal gap $g_{N}$ and its variation $\delta g_{N}$, the normal multiplier _... is not an additional unknown of the system to be solved. It is updated at every load step and calculated from $g_{N}$, by the expression (80). The linearization of $C_{N}$ is presented by:

$$
\begin{aligned}
& \frac{\partial c_{N}}{\partial \lambda_{N}}=0, \quad \frac{\partial c_{N}}{\partial g_{N}}=\varepsilon_{N} \delta g_{N}, \frac{\partial c_{N}}{\partial \delta g_{N}}=\bar{\lambda}_{N}+\varepsilon_{N} g_{N} z \\
& \text { And } \\
& \Delta c_{N}=\varepsilon_{N} \delta g_{N} \Delta g_{N}+\left(\bar{\lambda}_{N}+\varepsilon_{N} g_{N}\right) \Delta \delta g_{N}
\end{aligned}
$$

The value of the Lagrange multiplier presented in (81) is equals to

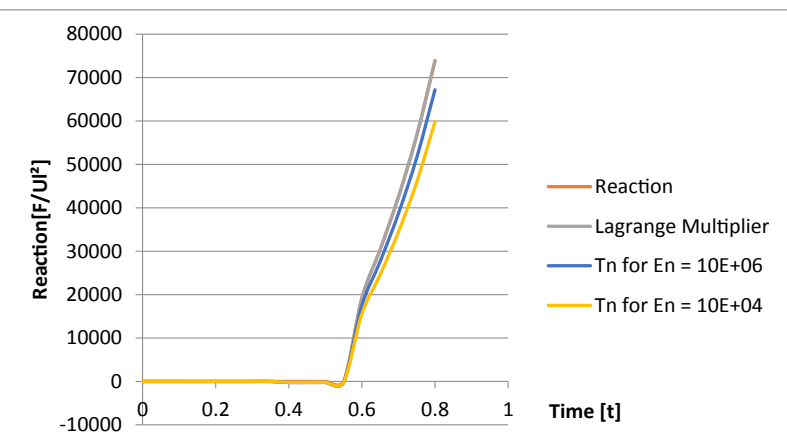

Figure 18: Evolution of the reaction and the Lagrange multipliers for the problem of contact between a disk and a basis. 


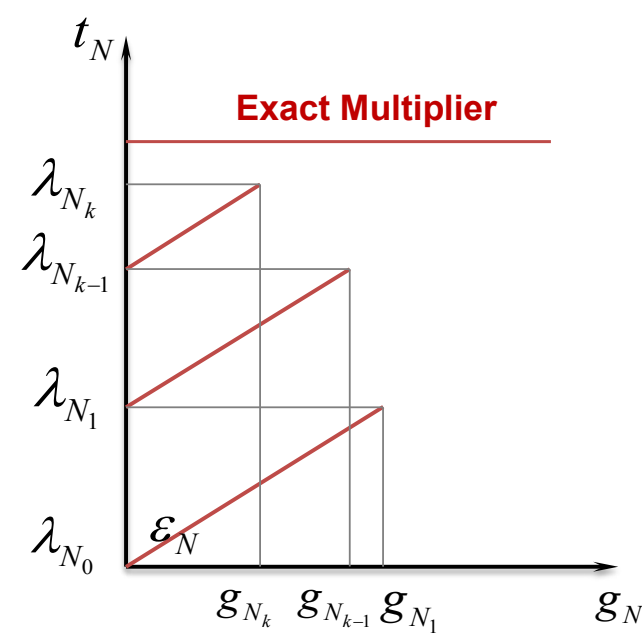

Figure 19: Scheme of Uzawa algorithm for multiplier augmentation.

the contact pressure. The tangent matrix and the residual vector for the augmented Lagrange formulation will be detailed in the section which follows.

\section{Expressions of the Residual Vector and the Tangent Matrix}

We resume the expression of the gap in (2.13) and we write it under matrix shape in the case of the augmented Lagrange formulation:

$$
g_{N}=\left\langle x_{n}^{A u g}\right\rangle\left[B_{G P}^{A u g}\right]\left\{n^{m}\right\}
$$

Multipliers used in this approach are not additional variables: thus the vector $\left\langle x_{n}^{A u g}\right\rangle$ and the shape function matrix $\left[B_{G P}^{A u g}\right]$ are identical to those used in the penalty formulation and detailed in (2) and (43). The segmentation of the contact interface in this approach is the same us used for the penalty formulation (Figure 2). The expression of the variation of (2.58) under matrix shape is identical to that defined in (2.19).

We can rewrite (2.55) as follows:

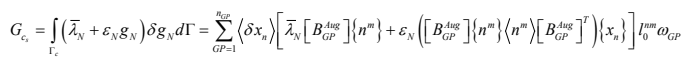

The contribution of integration point GP (Gauss Point) to the residual vector for the contact can be written in the case of augmented Lagrange formulation as follows:

$$
R_{N}^{A u g}=\left[\bar{\lambda}_{N}\left[B_{G P}^{A u g}\right]\left\{n^{m}\right\}+\varepsilon_{N}\left(\left[B_{G P}^{A u g}\right]\left\{n^{m}\right\}\left\langle n^{m}\right\rangle\left[B_{G P}^{A u g}\right]^{T}\right)\left\{x_{n}\right\}\right] l_{0}^{n m} \omega_{G P}
$$

We notice in the residual vector expression that if $\bar{\lambda}_{N}$ is zero, we find the expression of the residual vector of the penalty formulation. The variation of the natural coordinate $\xi$ is detailed in (49) and its linearization in (50). The linearization of the variation of the gap $\Delta$ $\left(\delta g_{\mathrm{N}}\right)$ is given in (56). To obtain the expression of the tangent matrix, it is necessary to linearize both terms of (83) by using (2.43) and (84).

Under matrix form we can rewrite (2.57) as follows:

$$
\Delta\left(c_{N}\right)=\left\langle\delta x_{n}\right\rangle\left[K_{N}^{\text {Aug }}\right]\left\{\Delta x_{n}\right\}
$$

The tangent matrix for the augmented Lagrange formulation is given by:

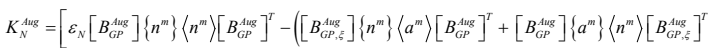

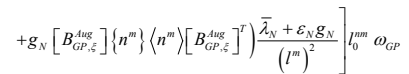

The matrix containing the derivatives of the shape functions $\left[B_{G P, \xi}\right]$ is identical to that used for the penalty formulation (60). The residual vector (86) and the tangent matrix (88) constitute the contribution of an integration point in the contact interface to the global system. Let us note that it is necessary to take into account the contribution of all the integration points of the interface.

\section{Algorithm of Resolution}

The significant difference of the augmented Lagrange method with regard to two other previously presented methods is that all the elements of the contact interface contribute to the virtual work of the system even if the normal gap is different from zero and thus the segment is inactive. This fact guarantees the regularity of the potential energy and the continuity of the virtual work. However, the inactive contact elements increase in a significant way the number of conditioning of the tangent matrix of the system. The phase of detection of the actual zone of contact must be carefully evoked for a resolution by the augmented Lagrange method.

To optimize the resolution of the contact by this formulation and reduce the conditioning of the tangent matrix, we are going to apply the algorithm of the active set deployed in the penalty method (Figure 4). And to keep the advantage of continuity of the virtual work, the nearby integration points of the points in the extremities are added to the conditioning of the contact. A detailed algorithm of resolution for the augmented Lagrange method is presented in the Figure 20.

If the problem converges for a load step (n), we keep the value of the multiplier $\lambda_{(k)}^{(n)}$ resulting from this calculation and we use it as initial multiplier in the augmentation algorithm in the following load step. This operation saves us useless iterations of augmentation seen that the exact value of the multiplier in the load step $(n+1)$ will be close to that in the previous load step.

By comparing the algorithm of resolution using the augmented Lagrange approach with that of the penalty formulation, we notice that the convergence of the global system in this approach is not sufficient to give a solution. The obtained solution has to pass a penetration test. And if the latter does not pass, we increase the value of the Lagrange multiplier used for the resolution and we begin again the calculation. With this technique, even with a low value of penalty coefficient, we do not risk to violate the non-penetration condition at the end of the load step. It is not the case with the penalty method.

\section{Example of Validation}

A ring is initially in contact with a beam, Figure 21. A hyper-elastic behavior with the Néo-Hooke law is considered for this problem [29]. The module of Young and the coefficient of Poisson for the ring as well as for the beam are indicated in the plan which follows. We apply a displacement in the vertical direction at the right extremity of the ring while the left side is embedded. Simultaneously, a horizontal displacement is applied to both extremities of the beam.

We use an augmented Lagrange formulation with a penalty coefficient $\varepsilon_{N}=5 \times 10^{3} \mathrm{~F} / U L^{2}$. The calculation is made for a period of time $[0,8 \mathrm{UT}]$ with a step of time $\Delta \mathrm{t}=0.025$ 


\section{Materials characteristics}

$E_{\text {ring }} 2.25 \times 10^{3} \mathrm{~F} / U L^{2}$

$v_{\text {ring }}=0.125$

$E_{\text {beam }} 2.7 \times 10^{3} \mathrm{~F} / \mathrm{UL}^{2}$

$v_{\text {beam }}=0.35$

Loading:

$\Delta_{x=} 16 U L$

$\Delta_{y=} 9.6 U L$

This example was also solved by the Lagrange multiplier and the penalty methods, a time step of $\Delta t=0.0125$ was necessary to solve the problem by means of the latter formulations. Generally, the augmented Lagrange method allows the resolution with a load step more important than the other formulations.

\section{Frictional Contact}

The contribution of the normal contact $C_{N}$ in the problem solution

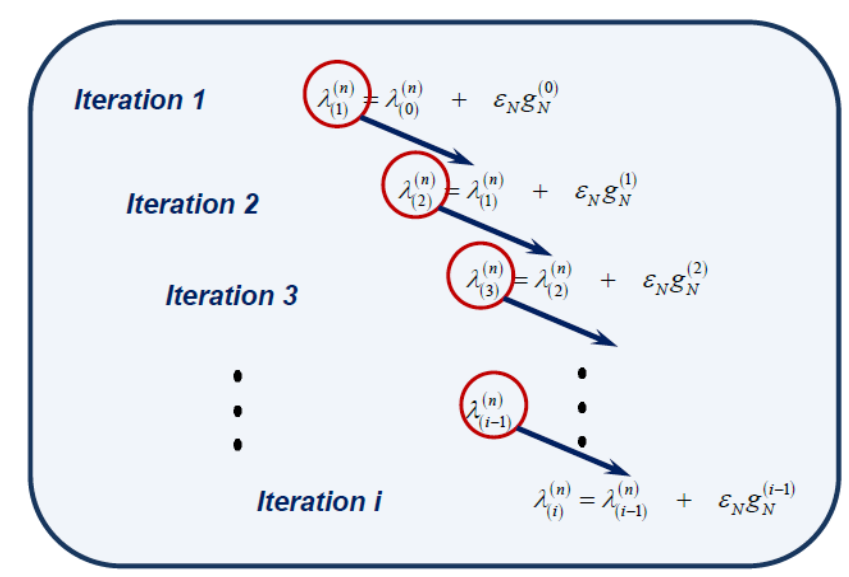

Figure 20: Scheme of the normal Lagrange multiplier augmentation on the load step'n'.

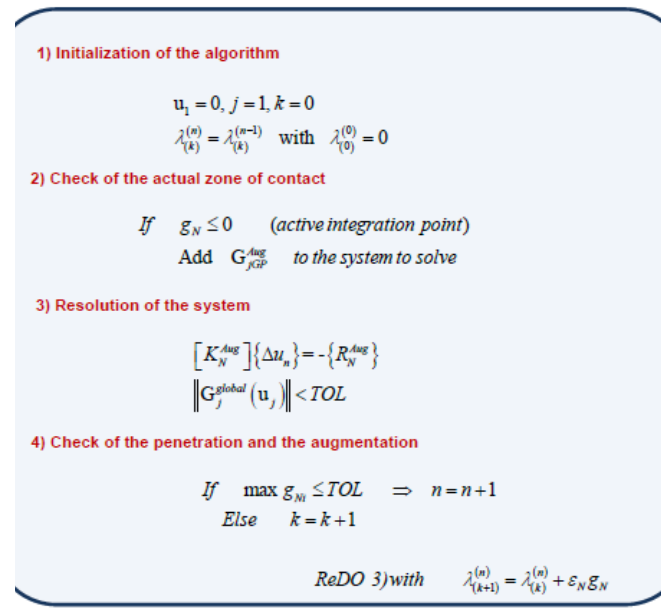

Figure 21: Algorithm of resolution of a contact problem of with the augmented Lagrange method. was already detailed in the previous section. It remains now to give the contribution of the tangent contact $C_{T}$ for the frictional problem. The expression of the virtual work of in the contact problem can be written as follows:

$$
\mathbf{t}_{c}=\mathbf{t}_{c_{N}}+\mathbf{t}_{c_{T}} z
$$

Knowing that the contact effort $t$ is the sum of the normal effort vector and tangent effort vector $\mathbf{t}_{c}=\mathbf{t}_{c_{N}}+\mathbf{t}_{c_{T}} z$. The expression of the latter varies according to the choice of the formulation.

\section{Penalty Method}

The use of the Newton-Raphson method for the problem resolution requires the linearization of the virtual work of contact contribution. With the penalty method, the contact effort can be described as follows:

$$
\mathrm{t}=\varepsilon \mathrm{g}=\varepsilon\left[x^{n m}-x^{m}(\xi)\right]
$$

When $\varepsilon$, is the penalty coefficient.

The expression of the contact virtual work for the penalty formulation is:

$$
G_{c_{s}}=\int_{\Gamma_{s}} \varepsilon\left[x^{n m}-x^{m}(\xi)\right]\left[\delta x^{n m}-\delta x^{m}(\xi)\right] d \Gamma
$$

For the linearization of (91), it is necessary to check the status (sliding or no). For stick contact, the natural coordinate value $\xi$ for a load step between two increments $t_{n}$ and $t_{n+1}$ does not change because the integration point does not leave the cone $\mu\left|p_{N}\right|$. We can neglect $\xi$ for the linearization because $\xi_{n=} \xi_{n+1}$. Using $x^{n n}\left(X^{n m}, t\right)=X^{n m}+u^{n m}\left(X^{n m}, t\right)$ and $x^{n m}\left(X^{n m}, t\right)=X^{n m}+u^{n m}\left(X^{n n}, t\right)$ one can rewrite (91) for a stick contact as follows:

$$
G_{c_{s}}^{\text {stick }}=\int_{\Gamma_{c}} \varepsilon\left[\delta u^{n m}-\delta u^{m}(\xi)\right] \cdot\left[\Delta u^{n m}-\Delta u^{m}(\xi)\right] d \Gamma
$$

For a sliding contact status, the linearization of (2.65) gives:

$$
\begin{aligned}
G_{c_{s}}^{s l i p}=\int_{\Gamma_{c}} & -\delta u_{, \xi}^{m} \Delta \xi \mathbf{t} d \Gamma+\varepsilon \int_{\Gamma_{c}}\left[\delta u^{n m}-\delta u^{m}(\xi)\right] \cdot\left[\Delta u^{n m}-\Delta u^{m}(\xi)\right] d \Gamma \\
& -\varepsilon \int_{\Gamma_{c}}\left[\delta u^{n m}-\delta u^{m}(\xi)\right] \cdot a^{m}(\xi) \Delta \xi d \Gamma
\end{aligned}
$$

The tangent matrix and the vector residue for the formulation of penalty for a problem of contact with friction will be detailed in the section which follows.

\section{Expressions of the Residual Vector and the Tangent Ma- trix}

By resuming the linearization of the virtual work for stick frictional contact, the mortar and non-mortar point vector (41) and the shape functions matrix (43), we can rewrite (92) as follows:

$$
R_{P e n}^{\text {stick }}=\varepsilon\left[B_{G P}\right]\left[B_{G P}\right]^{T}\left\{x_{n}\right\} l_{0}^{n m} \omega_{G P}
$$

The residual vector for stick frictional contact can be written with the penalty formulation as follows:

$$
R_{P e n}^{\text {stick }}=\varepsilon\left[B_{G P}\right]\left[B_{G P}\right]^{T}\left\{x_{n}\right\} l_{0}^{n m} \omega_{G P}
$$

If the integration point slides and leaves the friction cone, it is necessary to rewrite the virtual work (94) with the new position of $\xi_{\Sigma} \xi_{n+1}$ obtained by the projection outside of the friction cone which gives:

$$
G_{c_{s}}^{s l i p}=\varepsilon_{N} \sum_{G P=1}^{n_{G P}}\left[\left[B_{G P}\left(\xi_{n+1}\right)\right]\left[B_{G P}\left(\xi_{n+1}\right)\right]^{T}\left\{x_{n}\left(\xi_{n+1}\right)\right\}\right] l_{0}^{n m} \omega_{G P}
$$

And the residual vector: 


$$
R_{P e n}^{s l i p}=\varepsilon\left[B_{G P}\left(\xi_{n+1}\right)\right]\left[B_{G P}\left(\xi_{n+1}\right)\right]^{T}\left\{x_{n}\left(\xi_{n+1}\right)\right\} l_{0}^{n m} \omega_{G P}
$$

To determine the tangent matrix, it is necessary to linearize the variation of the contact virtual work expression. For stick frictional contact, we can neglect the variation of $\xi$. It is not the case if the integration point leaves the friction cone of and a sliding is noticed. In what follows let us be interested of stick contact.

$$
K_{P e n}^{\text {stick }}=\varepsilon\left[B_{G P}\right]\left[B_{G P}\right]^{T} l_{0}^{n m} \omega_{G P}
$$

We resume the gap for slip contact, when the sliding function $f_{s}=\| t_{c_{r}}|-\mu| t_{c_{v}} \mid$ nullifies, the new value of $\xi_{n+1}$ has to replace $\xi_{n}$ in (43) and (60). By using (94) and because the penalty coefficient value is the same for the normal and tangent direction, the expression sliding function in the load step $t_{n+1}$ can be replaced by:

$$
g_{T}\left(\xi_{n+1}\right) \operatorname{sign}\left(g_{T}\left(\xi_{n+1}\right)\right)-\mu g_{N}\left(\xi_{n+1}\right) \operatorname{sign}\left(g_{N}\left(\xi_{n+1}\right)\right)=0
$$

In (99) the sign can be negative as positive. The linearization of the natural coordinate can be obtained by the scalar product of (99) and the vector $a^{m}$ :

$$
\begin{aligned}
\Delta \xi= & -\frac{1}{\operatorname{sign}\left(g_{T}\right)\left\|a^{m}\right\|^{2}}\left[\left(\Delta u^{m m}-\Delta u^{m}\right) \cdot \operatorname{sign}\left(g_{\tau}\right) a^{m}-\mu \operatorname{sign}\left(g_{N}\right) \cdot n^{m}\left\|a^{m}\right\|\right. \\
& \left.+\left(x^{m m}-x^{m}\right) \cdot\left[\operatorname{sign}\left(g_{T}\right) \Delta u_{l_{\xi}^{m}}^{m}+\mu \operatorname{sign}\left(g_{N}\right) \frac{a^{m}}{\left\|a^{m}\right\|} \Delta u_{\zeta \xi}^{m} \cdot n^{m}-\mu \operatorname{sign}\left(g_{N}\right) \frac{n^{m}}{\left\|a^{m}\right\|} \Delta u_{\zeta \xi}^{m} \cdot a^{m}\right]\right]
\end{aligned}
$$

The tangent matrix for a slip contact is defined by:

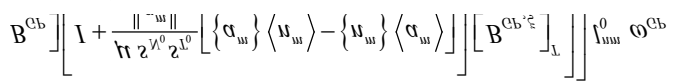

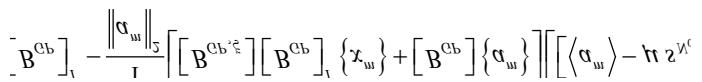

With

$$
\begin{aligned}
& s_{N_{0}}=\operatorname{sign}\left[\left\langle x_{n}\left(\xi_{n}\right)\right\rangle\left[B_{G P}\left(\xi_{n}\right)\right]\left\{n^{m}\left(\xi_{n}\right)\right\}\right] \\
& s_{T_{0}}=\operatorname{sign}\left[\left\langle x_{n}\left(\xi_{n}\right)\right\rangle\left[B_{G P}\left(\xi_{n}\right)\right] \frac{a^{m}\left(\xi_{n}\right)}{\left\|a^{m}\left(\xi_{n}\right)\right\|}\right]
\end{aligned}
$$

For a stick frictional contact problem, the residual vector (95) and the tangent matrix (98) allow to calculate the contribution of an integration point of the contact interface to the global system whereas for a slip frictional contact problem it is rather necessary to use the residual vector (97) and the tangent matrix (101). Let us note that it is necessary to take into account the contribution of all the integration points of the interface.

\section{Algorithm of Resolution}

At every load step, the contact and friction constraints are considered only for the active integration points. For the load step $t_{n}$, every integration point of the non-mortar segment is connected with his counterpart on the master segment by a projection. The position of the projection defined by the natural coordinate $\xi_{n}$ is saved for the following load step $t_{n+1}$ to judge the slip or stick status of the frictional contact. As can be seen in the algorithm described in the Figures 22 and 23, the resolution of the system depends at first on the information about the status of contact which defines the choice of the tangent matrix and the residual vector.

For the frictional problem, we keep the history of the projection from one load step to the following one. Therefore, the resolution requires more memory with regard to the frictionless problem; furthermore, the sliding test at the beginning of every load step of slows down the algorithm of resolution.

\section{Example of Validation}

An elastic ring constituted by two layers of thickness $e=5 U L$ each and of different rigidity is supported against fixed rigid base (Figure 24). The internal layer of the ring is 100 times stiffer than the outside one. The material of both layers is hyper-elastic, it is described by the NéoHooke law. A friction coefficient of friction is considered in the contact interface between the ring and the base. The ring at the beginning is not in contact with the base but offset of 20 UL. We apply a displacement on the vertical direction on both extremities of the ring of a maximal value $d_{y_{\max }}=60 U L$.

We notice a low difference between the reaction to the applied effort and the contact effort (Figures 25 and 26). This gap is due to the tolerance of this formulation in the penetration according to the value of the penalty coefficient. This offset is less important in the formulation of the contact by the augmented Lagrange method.

\section{Augmented Lagrange Method}

The expression of the contribution of frictional contact to the virtual work for the augmented Lagrange formulation $[5,30]$ is defined by:

$$
G_{c_{s}}=\int_{\Gamma_{c}}\left[t_{N} n \cdot \delta u-\mathbf{t}_{T} \cdot \delta u_{T}\right] d \Gamma
$$

For frictional contact problem, the normal contact effort $t_{\mathrm{N}}$ and the of tangential contact effort $t_{T}$ are both defined by the sum of a Lagrange multiplier and a penalty term. $t_{N}$ keep the same definition as frictionless contact (80). The expression of the tangent effort $t_{T}$ depends on the status of the contact (slip or stick). By using the definition of the sliding function, we can describe the evolution of the tangent effort by:

$$
\dot{u}_{T}-\xi \frac{\partial}{\partial \mathbf{t}_{T}} f_{s}=\frac{1}{\varepsilon_{T}} \dot{\mathbf{t}}_{T}
$$

With $\dot{u}_{T}$ is the variation of the displacement in the contact plan between the times steps $t_{n}$ and $t_{n+1}$. Let's note that $\dot{u}_{T}=0$ if the contact is stick $\left(f_{s}<0\right)$. Its linearization is given by:

$$
\Delta u_{T}=u_{T_{n+1}}-u_{T_{n}}
$$

The linearization of the Lagrange term in the variation of the tangent effort is given by:

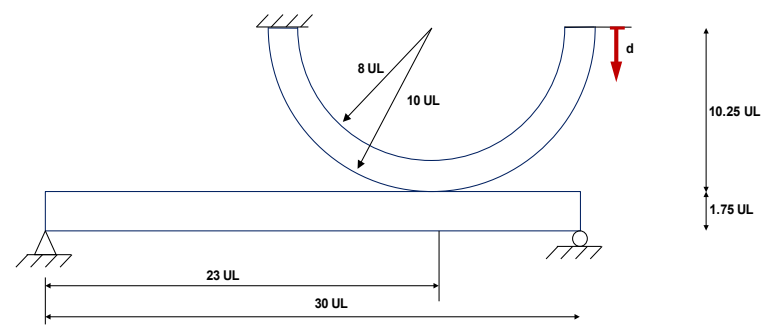

Figure 22: Data for the problem of contact between a ring and a beam.

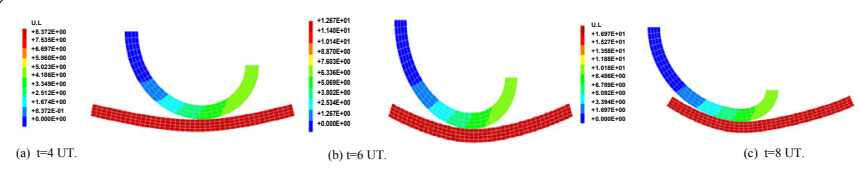

Figure 23: Displacement for the problem of contact between a ring and a beam. 
1) Initialization of the algorithm

$$
\mathrm{u}_{1}=0, j=1
$$

2) Check of the active contact zone

If $g_{N} \leq 0$ (active integration point)

Add $G_{j G P}^{P a}$ to the global system to solve

3) Test of Sliding and resolution of the system

If $\left\|\mathbf{t}_{c_{r}}\right\|-\mu\left|t_{c_{x}}\right|<0 \quad \Rightarrow$ no sliding

$\xi_{n+1}=\xi_{n}$

$\left[K_{P_{c n}}^{\text {sack }}\left(\xi_{n}\right)\right]\left\{\Delta u_{n}\right\}=-\left\{R_{P_{c n}}^{\text {suck }}\left(\xi_{n}\right)\right\}$

$\left\|\mathrm{G}_{j}^{\text {ghtal }}\left(\mathrm{u}_{j}\right)\right\|<T O L \Rightarrow S T O P$

else ||$t_{c_{r}} \|-\mu\left|t_{c_{N}}\right| \geq 0 \Rightarrow$ slipping

$\left[K_{P e n}^{\text {sip }}\left(\xi_{n+1}\right)\right]\left\{\Delta u_{n}\right\}=-\left\{R_{P e n}^{s k p}\left(\xi_{n+1}\right)\right\}$

$\left\|\mathrm{G}_{j}^{\text {global }}\left(\mathrm{u}_{j}\right)\right\|<T O L \Rightarrow S T O P$

Figure 24: Algorithm of resolution of a frictional contact problem by the penalty method.

$$
\Delta \lambda_{T}=\lambda_{T_{n+1}}-\lambda_{T_{n}}
$$

When an integration point changes its position in the contact surface, it is necessary to verify by applying the sliding function if it leaves the friction cone or not to define its contact status (stick or slip). We note the tangent effort calculated for the test of the status $t_{T_{n+1}}^{\text {test }}$, its expression is given by:

$$
\mathbf{t}_{T_{n+1}}^{\text {test }}=\mathbf{t}_{T_{n}}+\Delta \lambda_{T}+\varepsilon_{T} \Delta u_{T}
$$

It remains to give the expression of the linearization of the tangent effort variation:

$$
\begin{aligned}
& \Delta \xi \stackrel{\text { aianion: }}{\frac{f_{s_{n+1}}}{\varepsilon}} \text { if } f_{s_{n+1}}>0 \\
& \begin{array}{c}
\text { With } \\
\Delta \xi=\left\{\begin{array}{ccc}
0 & \text { if } & f_{s_{n+1}} \leq 0 \\
f_{s_{n+1}}
\end{array}=\frac{f_{s_{n+1}}}{\| \hat{\mathbf{t}}_{T_{n+1}}} \| \begin{array}{ll}
f_{s_{n+1}}>0 \\
-\mu & t_{N_{n+1}}
\end{array}\right.
\end{array}
\end{aligned}
$$

The vector $\mathbf{t}_{T_{n}}$ in the expression (109) is defined as the sum of the contribution of a penalty term and a Lagrange multiplier. Its represents the exact tangent effort after the convergence of the last load step at the time $t_{n}$. Given that the effort in the tangent plan (105) is defined by a time derivative, we use rather the term $\Delta \lambda_{\mathrm{T}}$ than the term $\lambda_{\mathrm{T}}$ for the tangent Lagrange multiplier. Using the definition of the tangent vector at the time step $t_{n}$, the expression of the contact virtual work (2.78) can be rewritten:

$$
G_{c_{s}}=\int_{\Gamma}\left[\left\langle\lambda_{N}+\varepsilon_{N} g\right\rangle \delta u \cdot n+\left(\delta \lambda_{T}+\varepsilon_{T} \delta u_{T}\right) \cdot \delta u_{T}\right] d \Gamma
$$

The contribution of an active integration point to the global solution passes by the calculation of its virtual work (2.86). The solution of the problem requires the use of the Newton-Raphson method. The tangent matrix and the residual vector for the augmented Lagrange formulation for a frictional contact problem will be detailed in the following section.

\section{Expressions of the Residual Vector and the Tangent Matrix}

By taking back the expression of the linearization of the frictional

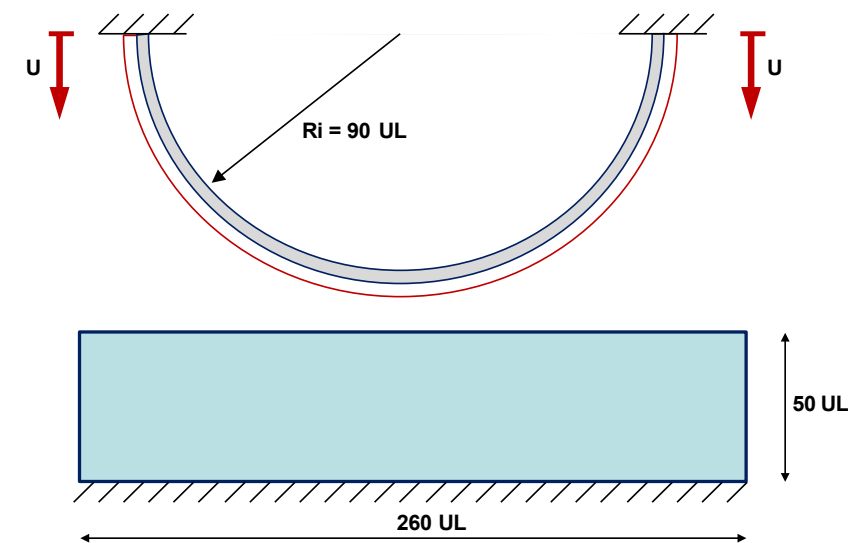

Figure 25: Contact problem between a ring and base.

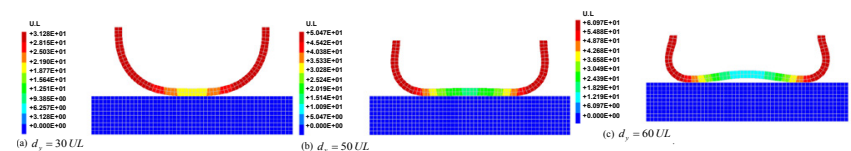

Figure 26: Displacement field for the contact problem between ring and base

contact virtual (112), the expression mortar and non-mortar points vector (41) and the of the shape functions matrix (43), we can write:

$$
\begin{aligned}
G_{c_{s}} & =\int_{\Gamma}\left[\left\langle\lambda_{N}+\varepsilon_{N} g\right\rangle \delta u \cdot n+\left(\delta \lambda_{T}+\varepsilon_{T} \delta u_{T}\right) \cdot \delta u_{T}\right] d \Gamma \\
& \left.=\sum_{G P=1}^{n_{G P}}\left\langle\delta x_{n}\right\rangle\left[\bar{\lambda}_{N}\left[B_{G P}\right]\left\{n^{m}\right\}+\lambda_{T}\left[B_{G P, \xi}\right]\left\{a^{m}\right\}+2 \varepsilon_{T}\left(\left[B_{G P}\right]\left[B_{G P}\right]^{T}\right)\left\{x_{n}\right\}\right]\right]_{0}^{l^{m m} \omega_{G P}}
\end{aligned}
$$

The residual vector for frictional contact can be written with the augmented Lagrange formulation as follows:

$$
\left.R_{\text {Aug }}^{\text {slipsich }}=\left[\bar{\lambda}_{N}\left[B_{G P}\right]\left\{n^{m}\right\}+\lambda_{T}\left[B_{G P, \xi}\right]\left\{a^{m}\right\}+2 \varepsilon_{T}\left(\left[B_{G P}\right]\left[B_{G P}\right]^{T}\right)\left\{x_{n}\right\}\right]\right]_{0}^{n m} \omega_{G P}
$$

Let us not forget that the calculation of the residual vector changes according to the status of contact (stick or slip). If the integration point slides by verifying the first inequality of the sliding function, the shape functions used in (114) will be calculated according to the new value of projection $\xi_{n+1}$. Otherwise, the natural coordinate keeps the same value obtained in the previous load step.

The tangent matrix for a frictional contact problem using the augmented Lagrange formulation can be decomposed into two parts: a part which represents the rigidity of contact for a normal effort and a second part for the rigidity to the tangential sliding. The matrix can be written as follows:

$$
K^{A u g}=K_{N}^{A u g}+K_{T}^{A u g}
$$

We are interested in what follows in the tangent part of the tangent matrix $K_{T}^{\text {Aug }}$. By applying the sliding function, we can meet two possible status of contact. One part of the matrix $K_{T}^{\text {Aug }}$ does not change expression independently of the status of the active integration point which is going to contribute to its rigidity. And a second part, noted $K_{T}^{\text {Aug sip sitick }}$, with the expression depends on the status of contact (stick or slip). We can write the tangent matrix as follows:

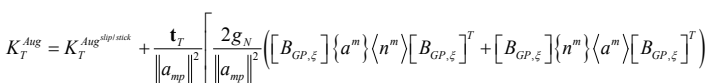

$$
\begin{aligned}
& +\frac{1}{\left\|a_{m p}\right\|^{2}}\left(\left[B_{G P, \xi}\right]\left\{a^{m}\right\}\left\langle a^{m}\right\rangle\left[B_{G P}\right]+\left[B_{G P}\right]\left\{a^{m}\right\}\left\langle a^{m}\right\rangle\left[B_{G P, \xi}\right]^{T}\right) \\
& \left.-\left[B_{G P}\right]\left\{n^{m}\right\}\left\langle n^{m}\right\rangle\left[B_{G P, \xi}\right]^{T}-\left[B_{G P, \xi}\right]\left\{n^{m}\right\rangle\left\langle n^{m}\right\rangle\left[B_{G P}\right]^{T}\right]
\end{aligned}
$$


As regards the first part we can note for the case of a stick contact:

$$
\begin{aligned}
K_{T}^{\text {Aug }} & =\frac{\varepsilon_{T}}{\left\|a_{m p}\right\|^{2}}\left[\left[B_{G P}\right]\left\{a^{m}\right\}\left\langle a^{m}\right\rangle\left[B_{G P}\right]^{T}+g_{N}\left[B_{G P}\right]\left\{a^{m}\right\}\left\langle n^{m}\right\rangle\left[B_{G P, \xi}\right]\right. \\
& +g_{N}\left[B_{G P, \xi}\right]\left\{n^{m}\right\}\left\langle a^{m}\right\rangle\left[B_{G P}\right]^{T}+\left(g_{N}\right)^{2}\left[B_{G P, \xi}\right]\left\{n^{m}\right\}\left\langle n^{m}\right\rangle\left[B_{G P, \xi}\right]^{T} \\
& \left.-2\left[B_{G P}\right]\left\{a^{m}\right\}\left\langle a^{m}\right\rangle\left[B_{G P, \xi}\right]^{T}-2\left[B_{G P, \xi}\right]\left\{n^{m}\right\}\left\langle a^{m}\right\rangle\left[B_{G P, \xi}\right]^{T}\right]
\end{aligned}
$$

And for the case of a slip status:

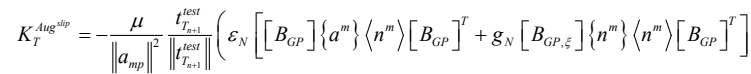

$$
\begin{aligned}
& \left.+t_{N}\left[\left[B_{G P}\right]\left\{a^{m}\right\}\left\langle a^{m}\right\rangle\left[B_{G P, \xi}\right]^{T}+g_{N}\left[B_{G P, \xi}\right]\left\{n^{m}\right\}\left\langle a^{m}\right\rangle\left[B_{G P, \xi}\right]^{T}\right]\right)
\end{aligned}
$$

For a stick frictional contact problem, the residual vector (114) and the tangent matrix described by (116) and (2.91), allow to calculate the contribution of an integration point on the contact interface to the global system and for a slip frictional contact problem it is rather necessary to use (118) instead of (117) for the tangent matrix and the same expression for the residual vector.

\section{Algorithm of Resolution}

To obtain the exact value of the multiplier of normal Lagrange ${ }_{N}$, we apply the augmentation formula (80) as for a frictionless problem. If the calculation converges in the load step $t_{n}$, we can deduct that the tangent multiplier is exactly equal to the tangent effort $\mathbf{t}_{T_{n+1}}=\lambda_{T_{n+1}}$. Also if the calculation converges in the load step $t_{n+1}$, we can write $\mathbf{t}_{T_{n+1}}=\lambda_{T_{n+1}}$. The term $\Delta \lambda_{\mathrm{T}}$ which presents the exact variation of the tangent multiplier between $t_{n}$ and $t_{n+1}$ is defined by:

$$
\mathbf{t}_{T_{n+1}}=\mathbf{t}_{T_{n}}+\Delta \lambda_{T}
$$

A frictional contact problem with augmented Lagrange method requires an augmentation algorithm for the normal multiplier $\lambda_{N}$ to find the exact value of the multiplier which represents the contact pressure (2.54), and an augmentation algorithm for the tangent multiplier ${ }_{T}$. By using (109) and (119), the augmented tangent multiplier can be defined by:

$$
\Delta \lambda_{T}^{(k+1)}=\Delta \lambda_{T}^{(k)}+\varepsilon_{T}\left(\Delta u_{T}^{(k)}-\Delta \xi^{(k)} \frac{\mathbf{t}_{T_{n+1}^{\text {test }}(k)}^{\text {tes }}}{\left.\left\|\mathbf{t}_{T_{n+1}}^{\text {tet }}\right\|\right)}\right)
$$

The terms in (2.94) with an index (k) are calculated in the load step $t_{n+1}$ but in the augmentation iteration $(\mathrm{k})$. As in the resolution of a frictionless problem, multipliers $\lambda_{N}$ and $\lambda_{T}$ are maintained constant during iteration. If the calculation does not converge, an augmentation will be necessary. From the augmentation iteration $(k)$ to $(k+1)$, the penalty decreases and the effort will be transferred to the Lagrange term until the convergence where the penalty term vanish and the Lagrange term will be exactly equal to the contact effort (Figure 27). As we have already do for the frictionless problem, if the solution converges for a load step (n), normal $\Delta \lambda_{t}^{(n+1)}$ and tangent $\Delta \lambda_{T}^{(n+1)}$ multipliers will not be initialized in zero at the beginning of the load step $(n+1)$ but rather by the values obtained after convergence of the previous load step (n) calculated in the last augmentation iteration.

\section{Example of Validation}

Let us consider the problem of frictional contact between a rectangular elastic block, dimension $(4 \mathrm{UL} \times 2 \mathrm{UL})$, putted down on a rigid frame [5,30-32]. We apply a pressure to the top of the block $P_{Y=} 200$ $F / U L^{2}$ and a surface horizontal effort $P_{X=} 60 \mathrm{~F} / U L^{2}$ on the right tip. The frame, considered stiff, is 100000 times stiffer than the block (Figure 27). This problem was already handled with the penalty formulation by Wriggers et al. in [31]. In the latter, the authors manage to find a solution for the problem in a single load step with Coulomb friction law. But they eliminated the first and the last knots of the block in the active contact zone.

Materials characteristics:

$$
\begin{aligned}
& E_{\text {block }}=1.10^{3} \mathrm{~F} / \mathrm{UL}^{2} \\
& E_{\text {frame }}=1.10^{8} \mathrm{~F} / \mathrm{UL}^{2} \\
& V_{\text {block }=} 0.3 \\
& V_{\text {frame }}=0.0 \\
& \text { Loading } \\
& P_{Y}=200 \mathrm{~F} / \mathrm{UL}^{2} \\
& P_{X}=60 \mathrm{~F} / \mathrm{UL}^{2}
\end{aligned}
$$

Augmented Lagrange parameters:

$$
\begin{aligned}
& \varepsilon_{N}=1 \times 10^{8} \mathrm{~F} / \mathrm{UL}^{2} \\
& \varepsilon_{T}=1 \times 10^{4} \mathrm{~F} / U L^{2}
\end{aligned}
$$

Coefficient of friction:

$$
\mu=0.5
$$

\section{Numerical Examples}

All the numerical examples are calculated using FiEStA code. It's a two-dimensional finite element house code.

\section{Ironing problem}

In the first step the disk moves vertically downward with $\mathrm{w}=0,72$ $\mathrm{UL}$, then it translate horizontally with $\mathrm{u}=9,6 \mathrm{UL}$ while maintaining the vertical displacement of the first step. The displacements are applied on the upper half of the disk (Figure 28). The block and the disc both have a hyper-elastic behavior (with Néo-Hooke law). The disk modulus Young is $E_{\text {disk }}=6.896 \times 10^{3} \mathrm{~F} / \mathrm{UL}^{2}$, and it's 10 times stiffer than the block but they have the same Poisson coefficient $v=0.35$. We use a penalty formulation which the coefficient is $\varepsilon_{\mathrm{N}=} 5 \times 10^{3} \mathrm{~F} / U L^{2}$. We can refer for this problem to [9].

\section{Simulation of drawbeads in sheet metal forming}

In many complex operations of metal forming, the blank deformation is generally controlled by drawbeads (Figure 29). For more details of this example we can refer to [33-35]. The geometrical data are presented in (Figure 30). The Young modulus and the Poisson coefficient for the sheet (aluminum AL60022-T4) are $E_{\mathrm{AL} 60022-\mathrm{T} 4=} 68 \mathrm{Gpa}$ and $V_{\mathrm{AL} 60022-\mathrm{T} 4=} 0.29$. The punch and the matrix are on high-strength steel which its Young modulus and the Poisson coefficient are $E_{\mathrm{HSS}=} 200 \mathrm{Gpa}$ and $v_{\mathrm{HSS}}=0.3$. The plasticity was not considered in this case, our main concern is the modelling of the contact.

\section{Press-fit problem}

This problem presents the simulation of a rectangular rubber block pressed through a metallic channel (Figures 31-37). The block has a hyper-elastic behavior (Néo-Hooke law) and the steel channel has an elastic behavior [28]. The Young modulus and the Poisson coefficient for the rubber block are $E_{\text {rubber }}=1 \times 10^{9} \mathrm{Mpa}$ and $v_{\text {rubber }}=0.43$. The channel is steel which the Young Modulus is $E_{\text {steel }}=210 \times 10^{9} \mathrm{Mpa}$ and the Poisson coefficient is $v_{\text {steel }} 0.3$. The rubber block moves in the horizontal direction with $\Delta u_{\max }=130 \mathrm{~mm}$. We modelled half of the problem by 
Citation: Kallel A, Bouabdallah S (2017) 2d Frictional B-Spline Smoothed Mortar Contact Problems Part II: Resolution Phase. J Appl Mech Eng 6: 275. doi: $10.4172 / 2168-9873.1000275$

Page 15 of 18

taking into account symmetry conditions.

The simulation of the press-fit problem of a rubber block in a steel channel becomes possible with smoothing. The section reducing of the channel in the middle presents a singularity in the normal vector continuity. This singularity is reduced thanks to the smoothing.

\section{Conclusion}

The choice of the formulation for the application of the contact and friction constraints has a large influence on the quality of the obtained result. Although the penalty method presents the formulation the most used in the resolution of the contact problems, the solution remains too much depend on the choice of the penalty coefficient. In spite of the research effort for the automation of the value of this coefficient, its influence on the quality of the calculation is decreased but not eliminated. The Lagrange multipliers method presents an exact solution and not an approximation as in the case of penalty formulation. This

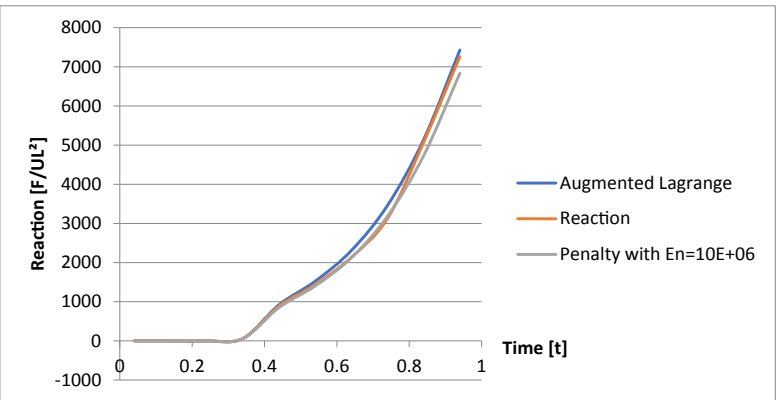

Figure 27: Evolution of the reaction and the contact effort for the problem of contact between ring and base.

$$
\begin{aligned}
& \text { 1) Initialization of the algorithm } \\
& \mathrm{u}_{1}=0, j=1, k=0 \\
& \lambda_{N}^{(0)}=\left\langle\lambda_{N}+\varepsilon_{N} g_{N}\right\rangle \text { et } \Delta \lambda_{T}^{(0)}=0 \\
& \text { 2) Check of the active contact zone } \\
& \text { If } g_{N} \leq 0 \text { (actif integration point) } \\
& \text { Add } \mathrm{G}_{F(\mathrm{P})}^{\mathrm{Pa}} \text { to the system to soive } \\
& \text { 3) Test of Sliding and resolution of the system } \\
& \text { If } \| t_{{ }_{\sigma}}||-\mu\left|t_{c_{\alpha}}\right|<0 \Rightarrow \text { no slipping } \\
& {\left[K^{\text {Aug }}\left(\xi_{n}\right)\right]\left\{\Delta u_{n}\right\}=-\left\{R_{\text {Aug }}^{\text {s.m/ Kuscol }}\left(\xi_{n}\right)\right\}} \\
& \text { If }\left\|t_{\alpha}\right\|-\mu\left|t_{c \mathrm{cv}}\right| \geq 0 \Rightarrow \text { slipping } \\
& {\left[K^{\text {Aug }}\left(\xi_{n+1}\right)\right]\left\{\Delta u_{n}\right\}=-\left\{R_{\text {Ang }}^{\text {ipitind }}\left(\xi_{n+1}\right)\right\}} \\
& \text { 4) Augmentation of multipliers } \\
& \text { If } g\left(u_{n+1}^{(k)}\right) \leq \text { TOL1 And }\left\|u_{\tau_{T_{m}}}-u_{\tau_{n}}\right\| \leq \text { TOL2 } \Rightarrow n=n+1 \\
& \text { Else } k=k+1 \quad \text { And Go To 3) with } \\
& \lambda_{N}^{(k+1)}=\left\langle\lambda_{N}^{(k)}+\varepsilon_{N} g\left(u_{n+1}^{(2)}\right)\right\rangle \\
& \int \Delta \lambda_{T}^{(k)}+\varepsilon_{\tau} \Delta u_{T}^{(k)} \quad 0 \quad \text { if stick }
\end{aligned}
$$

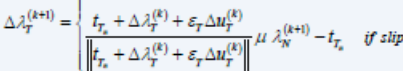

Figure 28: Algorithm of resolution of frictional contact problem with augmented Lagrange method. method presents an almost no tolerance to the penetration what returns the calculation sometimes difficult to converge in certain applications. Besides, the Lagrange multipliers, which present the contact efforts, are considered as additional unknowns to calculate in the global solution. In the literature, we find works for the condensation of these multipliers to avoid the increase of the problem size, but this operation presents an additional effort to the resolution. These observations motivated us to use the augmented Lagrange formulation. This method is very present in the literature for the resolution of the frictional contact problems. It offers a solution with a good precision close to that proposed by the Lagrange multipliers formulation without adding unknowns to the system to be solved. The choice of the penalty coefficient is low compared with the penalty method. The description of the terms necessary for the implementation of this formulation by finite elements method is almost absent in the literature contrary in two other formulations. In this work we tried to present detailed expressions of the terms necessary for the implementation and also a detailed resolution algorithm for both frictionless and frictional contact.

The modelling of the contact illustrated in this work is restricted to the two-dimensional quasi-static problems. For a simulation of a stamping problem, an extension in the three-dimensional domain will be essential. The necessary terms for the implementation in FiEStA are with implicit scheme. This work can be generalized by dynamics contact to solve more problems [36]. The modelling of dynamics contact problem can be developed either with implicit or explicit scheme.

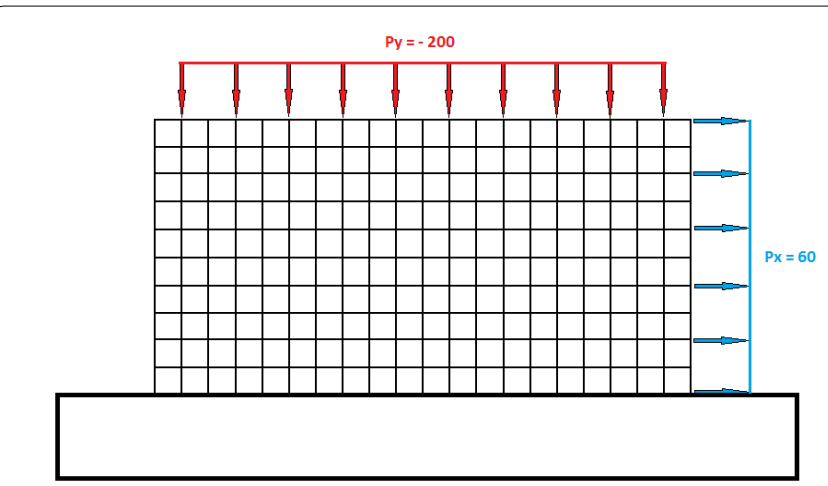

Figure 29: Frictional contact problem between a deformable block and a rigid frame.

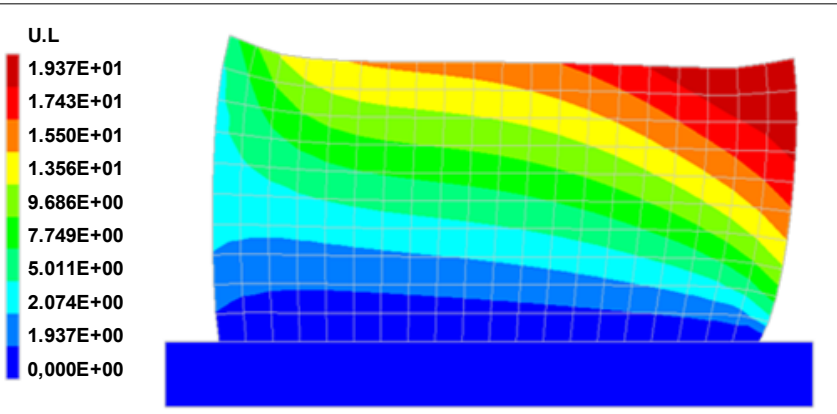

Figure 30: Displacement filed of a frictional contact problem between a deformable block and a rigid frame. 
Citation: Kallel A, Bouabdallah S (2017) 2d Frictional B-Spline Smoothed Mortar Contact Problems Part II: Resolution Phase. J Appl Mech Eng 6: 275. doi: 10.4172/2168-9873.1000275

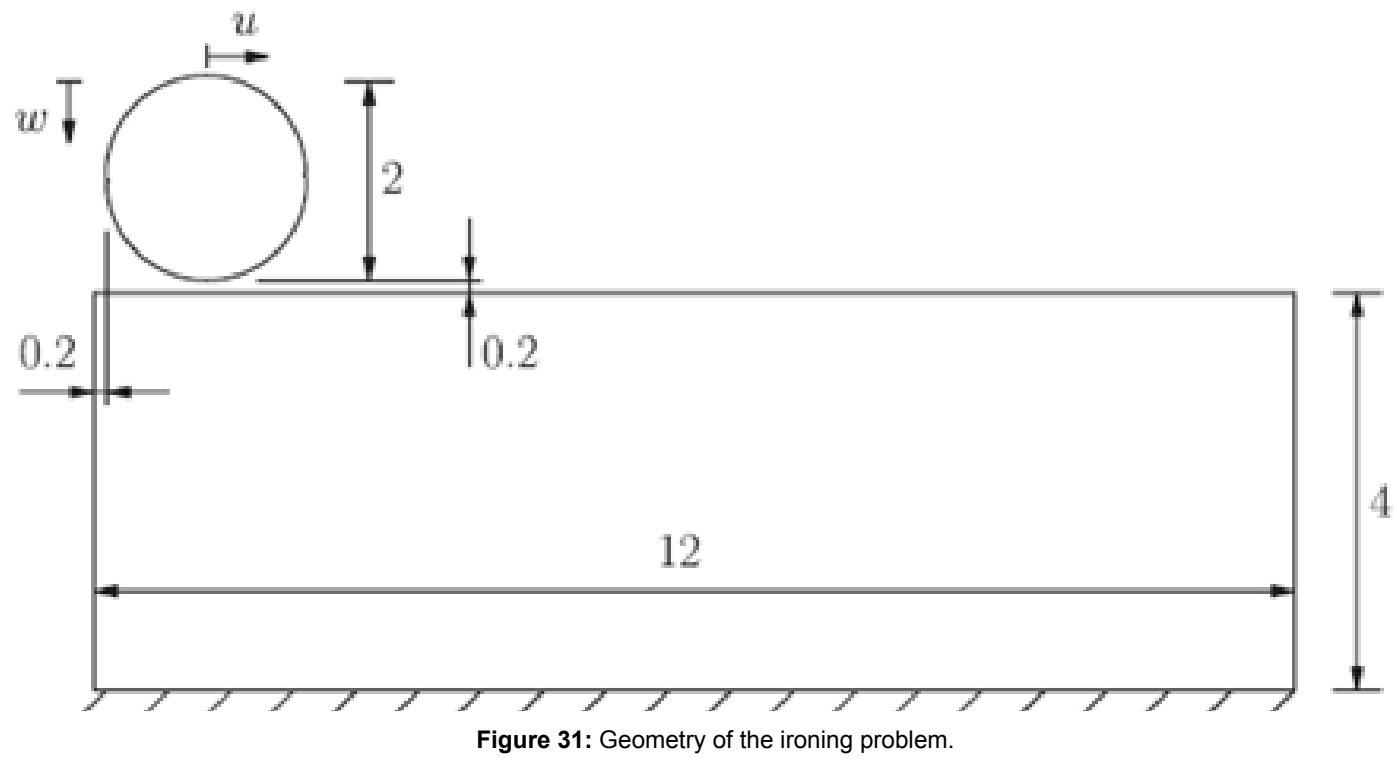

U.

$-5.453 E-02$

$-6.709 \mathrm{E}-02$

$-1.396 \mathrm{E}-01$

$-2.122 E-01$

$-2.847 \mathrm{E}-01$

$-3.573 \mathrm{E}-01$

$-4.298 \mathrm{E}-01$

$-5.026 \mathrm{E}-01$

$-5.742 E-01$

$-6.475 E-01$

-7.200 E-01

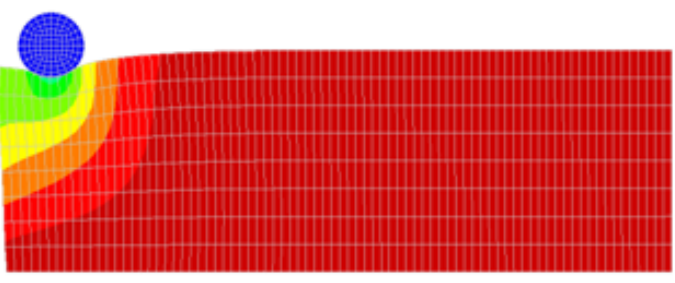

a)

U.L

$+1.086 \mathrm{E}-02$

$-6.222 \mathrm{E}-02$

$-1.353 \mathrm{E}-01$

$-2.084 \mathrm{E}-01$

$-2.815 \mathrm{E}-01$

$-3.546 \mathrm{E}-01$

$-4.277 \mathrm{E}-01$

$-5.007 \mathrm{E}-01$

$-5.738 \mathrm{E}-01$

$-6.469 \mathrm{E}-01$

$-7.200 \mathrm{E}-01$

gure 31: Geometry of the ironing problem.

$-5.153 E-02$

$-5.153 \mathrm{E}-02$

$6.736 \mathrm{E}-02$

$-1.399 \mathrm{E}-01$

$-2.124 \mathrm{E}-01$

-2.849E-01

$-3.574 \mathrm{E}-01$
$-4.299 \mathrm{E}-01$
$-5.025 E-01$

$-4.299 \mathrm{E}-01$

$6.475 \mathrm{E}-01$

$-7.200 \mathrm{E}-01$

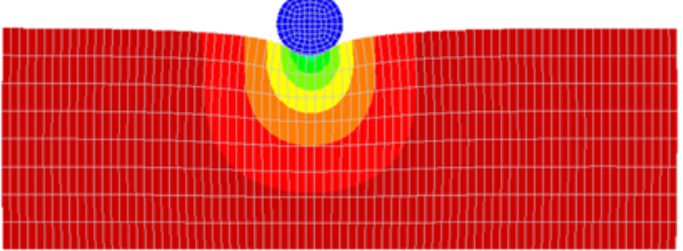

(b)

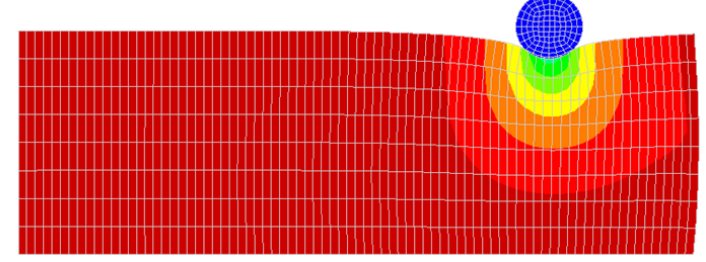

(c)

Figure 32: Displacement field of the ironing problem.(a) $u=0, w=0.72 U L$, (b) $u=4.8 U L, w=0.72 U L$, (c) $u=9.6 U L, w=0.72 U L$. 
Citation: Kallel A, Bouabdallah S (2017) 2d Frictional B-Spline Smoothed Mortar Contact Problems Part II: Resolution Phase. J Appl Mech Eng 6: 275. doi: $10.4172 / 2168-9873.1000275$

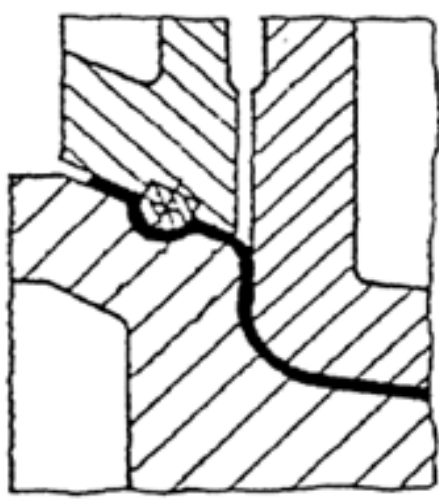

Figure 33: Use of the drawbeads in stamping.

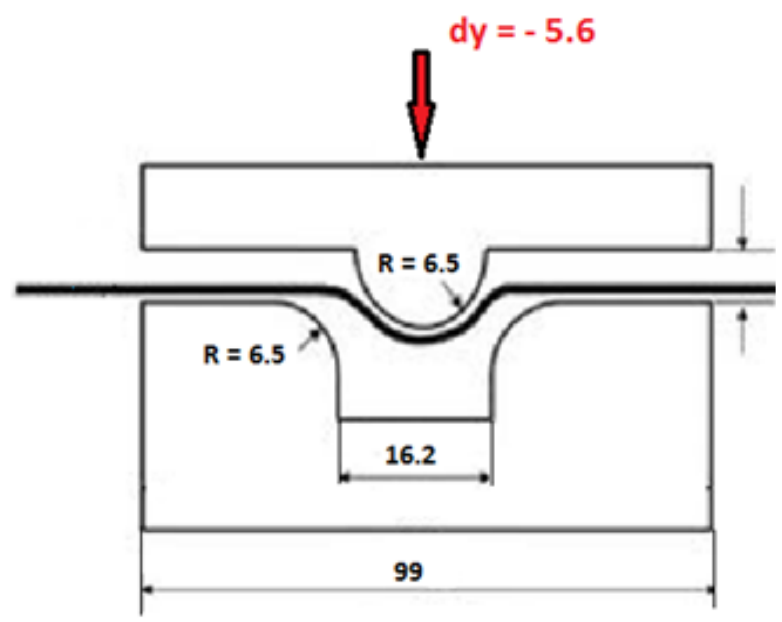

Figure 34: Geometrical data of the drawbeads.

$+5.652 E+00$

$+5.087 \mathrm{E}+00$

$+4.521 \mathrm{E}+00$

$+3.956 \mathrm{E}+00$

$+3.391 E+00$

$+2.826 \mathrm{E}+00$

$+2.261 E+00$

$+1.696 \mathrm{E}+00$

$+1.130 \mathrm{E}+00$

$+5.652 \mathrm{E}-01$

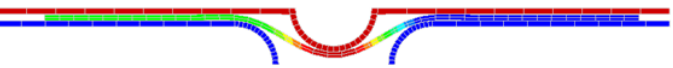

Figure 35: Displacement field of the drawbeads simulation.

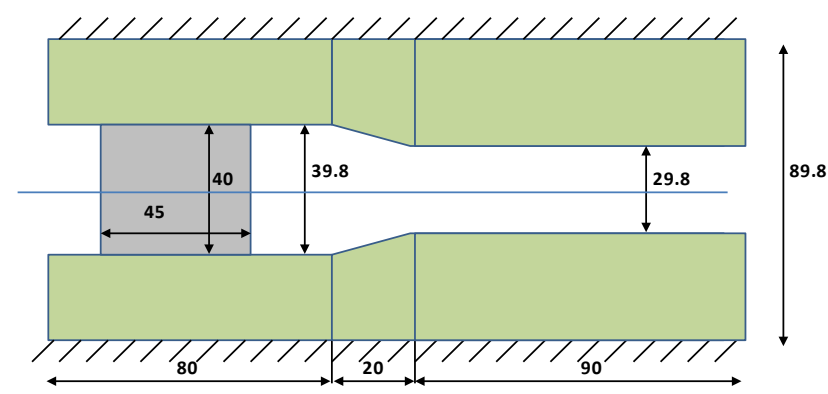

Figure 36: Geometrical data of the press-fit problem.
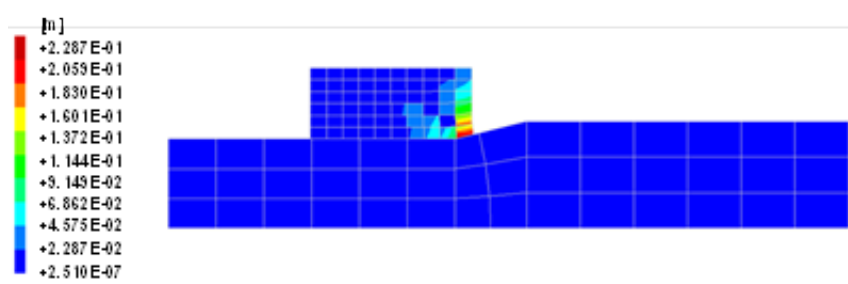

$2.287 \mathrm{E}-102$

(a) $u=30 \mathrm{~mm}$.

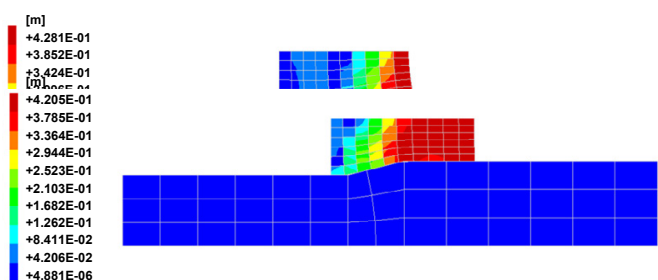

(c) $\mathrm{u}=70 \mathrm{~mm}$.

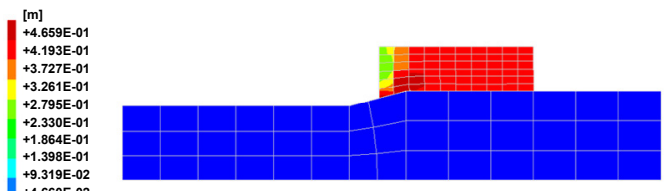

(d) $\mathrm{u}=90 \mathrm{~mm}$.

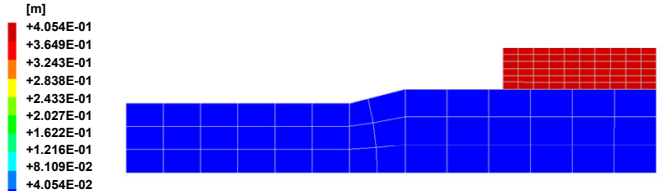

(e) $\mathrm{u}=130 \mathrm{~mm}$

Figure 37: Displacement field of the press-fit problem.

\section{Acknowledgement}

This research was conducted as part of the OASIS project, supported by OSEO within the contract FUI no. F1012003Z.

\section{References}

1. Simo JC, Wriggers $P$, Taylor RL (1985) A perturbed Lagrange formulation or the finite element solution of contact problems. Comp Methods in Appl Mech and Eng 50: 163-180.

2. Kikuchi N, Oden JT (1988) Contact problems in elasticity: A Study of Variational Inequalities and Finite Element Methods. SIAM, Philadelphia.

3. Bertsekas DP (1984) Constrained optimization and Lagrange multiplier Methods. Academic Press, New York.

4. Wriggers $P(2006)$ Computational contact mechanics. $2^{\text {nd }}$ edn, Springer-Verlag

5. Simo JC, Laursen TA (1992) An augmented lagrange treatment of contact problems involving friction. Computers and Structures 42: 97-116.

6. Hallquist JO (1979) Nike2d: An implicit, finite-deformation, finite element code for analysing the static and dynamic response of two-dimensional solids. University of California, Lawrence Livermore National Laboratory.

7. Rebel C, Park KC, Felippa CA (2000) A contact-impact formulation based on localized Lagrange multipliers. College of Engineering at University of Colorado Bolder, Colorado 80309.

8. Fischer KA, Wriggers $P(2005)$ Frictionless $2 d$ contact formulations for finite deformations based on the mortar method. Computational Mechanics 36 226-244. 
Citation: Kallel A, Bouabdallah S (2017) 2d Frictional B-Spline Smoothed Mortar Contact Problems Part II: Resolution Phase. J Appl Mech Eng 6: 275. doi: 10.4172/2168-9873.1000275

Page 18 of 18

9. Popp A, Gee MW, Wolfgang A Wall (2009) A finite deformation mortar contact formulation using a primal-dual active set strategy. Int $\mathrm{J}$ Numer Mech Eng 79 : 1354-1391.

10. Papadopoulos $P$, Taylor RL (1990) A mixed formulation for the finite element solution of contact problems. University of California at Berkeley.

11. Powell MJD (1969)A method for nonlinear constraints in minimization problems. Optimization, Academic Press, London.

12. Fletcher $R$ (1970) A class of methods in nonlinear programming with termination and convergence properties. Integer and Nonlinear Programming. NorthHolland Publishing Company.

13. Glowinski R, Le Tallec P (1984) Finite element analysis in nonlinear incompressible elasticity. In: Finite Element Special Problems in Solid Mechanics. Prentice-Hall, Englewood Cliffs, New Jersey.

14. Laursen TA, Simo JC (1993) Algorithmic symmetrisation of Coulomb frictional problems using augmented Lagranges. Comp Methods in Appl Mech and Eng 108: 133-146.

15. Pietrzak G, Curnier A (1999) Large deformation frictional contact mechanics: continuum formulation and augmented Lagrangean treatment. Methods in Appl Mech and Eng 177: 351-381.

16. Yastrebov V (2011) A Computational contact mechanics: geometry, detection and numerical techniques.

17. Luenberger DG (1984) Linear and nonlinear programming. Addison-Wesley Reading, Mass.

18. Zavarise G, De Lorenzis L, Taylor RL (2012) A non-consistent start-up procedure for contact problems with large load-steps. Computer Methods in Appl Mech and Eng 205: 91-109.

19. Wohlmuth $\mathrm{BI}$ (2000) A mortar finite element method using dual spaces for the Lagrange multiplier. SIAM, J Numerical Analysis 38: 989-1012.

20. Hueber S, Wohlmuth B (2005b) A primal-dual active set strategy for non-linear multibody contact problems. Comp Methods in Appl Mech and Eng 194: 31473166 .

21. Hueber S, Mair M, Wohlmuth B (2005a) A priori error estimates and an inexact primal dual active set strategy for linear and quadratic finite elements applied to multibody contact problems. Appl Numerical Math 54: 555-576.

22. Flemiscli B, Puso MA, Wolilmutli B (2005) A new dual mortar method for curved interfaces: 2d elasticity. Int J Numerical Methods in Eng 63: 813-832.

23. Hartmann S, Brunssen S, Ramm E, Wohlmuth B (2007) Unilateral non-linear dynamic contact of thin-walled structures using a primal-dual active set strategy Int J Numer Meth Eng 70: 883-912.

24. Hartmann S, Ramm E (2008) A mortar based contact formulation for non-linear dynamics using dual Lagrange multipliers. Finite Elements in Analysis and Design 44: $245-258$.

25. Popp A, Gitterle M, Gee MW, Wall WA (2010) A dual mortar approach for 3D finite deformation contact with consistent linearization. Int J Numerical Methods in Eng 83:1428-1465.

26. Cichosz T, Bischoff M (2011) Consitent treatment of bounderies with mortar contact formulations using dual Lagrange multipliers. Comp Methods in Appl Mech and Eng 200: 1317-1332.

27. Tur M, Giner E, Fuenmayor FJ, Wriggers P (2012) 2D contact smooth formulation based on the mortar method. Computer Methods in Appl Mech and Eng 247-248: 1-14.

28. Fischer KA, Wriggers $P$ (2006) Mortar based frictional contact formulation for higher order interpolations using the moving friction cone. Comp Methods in Appl Mech and Eng.

29. Yang B, Laursen TA, Meng X (2005) Two dimensional mortar contact methods for large deformation frictional sliding. Int J Numerical Methods in Eng 62: 1183-1225.

30. Heege A, Alart P (1996) A frictional contact element for strongly curved contact problems. Int J Numerical Methods in Eng 39: 165-184.

31. Wriggers P, Van vu T Stein E (1990) Finite element formulation of large deformation impact-contact problems with friction. Computers \& Structures 37 319-331.

32. Oden JT, Pires EB (1984) Algorithms and numerical results for finite element approximations of contact problems with non-classical friction laws. Comput. Struct 19: 137-147.

33. Padmanabhan V, Laursen TA (2001) A framework for development of surface smoothing procedures in large deformation frictional contact analysis. Finite Elements in Analysis and Design 37: 173-198.

34. Maker B, Samantha SK, Grab B, Triantafylidis N (1987) An analysis of drawbeads in sheet metal forming. Part II: Experimental verification. J Eng Mater Technol 109: 164-170.

35. Chun BK, Kim HY, Lee JK (2002) Modeling the bauschinger effect for sheet metals. part II: Applications. Int Jou Plas 18: 597-616.

36. Magnain B, Feng ZQ, Cro JM (2005) Modélisation des problèmes d'impact avec dissipation d'énergie par frottement. Compte-rendu Mécanique 333 : 419-424. 